\title{
Variant Signal Peptides of Vaccine Antigen, FHbp, Impair Processing Affecting Surface Localization and Antibody-Mediated Killing in Most Meningococcal Isolates
}

\author{
Ronni A. G. da Silva ${ }^{1}$, Andrey V. Karlyshev' ${ }^{2}$, Neil J. Oldfield' ${ }^{1}, K^{2}$ arl G. Wooldridge', \\ Christopher D. Bayliss ${ }^{3}$, Ali Ryan ${ }^{2}$ and Ruth Griffin ${ }^{1 *}$ \\ ${ }^{1}$ Centre for Biomolecular Sciences, University of Nottingham, Nottingham, United Kingdom, ${ }^{2}$ School of Life Sciences, \\ Pharmacy and Chemistry, Kingston University, Kingston upon Thames, United Kingdom, ${ }^{3}$ Department of Genetics \\ and Genome Biology, University of Leicester, Leicester, United Kingdom
}

OPEN ACCESS

Edited by:

Maria Laura Gennaro, Rutgers, The State University of

New Jersey, United States

Reviewed by:

William Doerrler,

Louisiana State University,

United States

Phil Giffard,

Charles Darwin University, Australia

*Correspondence:

Ruth Griffin

ruth.griffin1@nottingham.ac.uk

Specialty section:

This article was submitted to

Infectious Diseases,

a section of the journal

Frontiers in Microbiology

Received: 16 September 2019

Accepted: 25 November 2019

Published: 19 December 2019

Citation:

da Silva RAG, Karlyshev AV,

Oldfield NJ, Wooldridge KG,

Bayliss $C D$, Ryan $A$ and Griffin $R$ (2019) Variant Signal Peptides of

Vaccine Antigen, FHbp, Impair

Processing Affecting Surface Localization and Antibody-Mediated

Killing in Most Meningococcal Isolates. Front. Microbiol. 10:2847.

doi: 10.3389/fmicb.2019.02847
Meningococcal lipoprotein, Factor $\mathrm{H}$ binding protein ( $\mathrm{FHbp}$ ), is the sole antigen of the Trumenba vaccine (Pfizer) and one of four antigens of the Bexsero vaccine (GSK) targeting Neisseria meningitidis serogroup B isolates. Lipidation of FHbp is assumed to occur for all isolates. We show in the majority of a collection of United Kingdom isolates (1742/1895) non-synonymous single nucleotide polymorphisms (SNPs) in the signal peptide (SP) of FHbp. A single SNP, common to all, alters a polar amino acid that abolishes processing: lipidation and SP cleavage. Whilst some of the FHbp precursor is retained in the cytoplasm due to reduced binding to SecA, remarkably some is translocated and further surface-localized by Slam. Thus we show Slam is not lipoprotein-specific. In a panel of isolates tested, the overall reduced surface localization of the precursor FHbp, compared to isolates with an intact SP, corresponded with decreased susceptibility to antibody-mediated killing. Our findings shed new light on the canonical pathway for lipoprotein processing and translocation of important relevance for lipoprotein-based vaccines in development and in particular for Trumenba.

Keywords: meningoccocus, FHbp, vaccine, signal peptide, lipoprotein, Lnt, Slam

\section{INTRODUCTION}

Neisseria meningitidis is a leading cause of bacterial meningitis and sepsis with high fatality (up to $50 \%$ when untreated) and high frequency (more than 10\%) of severe sequelae (Rappuoli et al., 2018). Polysaccharide-based vaccines are effective in preventing disease caused by isolates of serogroups A, C, W, and Y but are ineffective against those of serogroup B (MenB) (Pace and Pollard, 2007). The lipoprotein, Factor $\mathrm{H}$ binding protein (FHbp), is a major virulence factor, which recruits human factor $\mathrm{H}(\mathrm{fH})$ to the meningococcal surface preventing complement from binding to the bacterium and thus inhibiting bacteriolysis by the alternative complement pathway (Schneider et al., 2006). The amino acid sequence of FHbp varies with identities as low as $60 \%$ between isolates which led to the classification of this lipoprotein into subfamily A (subdivided into variant groups 2 and 3) and subfamily B (variant group 1) (Masignani et al., 2003; Fletcher et al., 2004; 
Brehony et al., 2009; Jiang et al., 2010). Despite this variation, FHbp emerged as a promising vaccine candidate due to its ability to stimulate a strong serum bactericidal antibody (SBA) response capable of killing diverse group $\mathrm{B}$ isolates (Fletcher et al., 2004). It is thought that FHbp-specific antibodies not only promote bactericidal killing by the classical pathway but also via amplification of the alternative pathway, by preventing $\mathrm{fH}$ from binding to FHbp (Giuntini et al., 2011).

Lipoproteins, such as FHbp, are synthesized as precursors (preprolipoproteins) in the cytoplasm, which are subsequently taken through a sequential pathway for processing and sorting to the outer membrane (OM) (Kovacs-Simon et al., 2011; da Silva et al., 2017). The N-terminal signal peptide (SP), characteristic of bacterial lipoproteins, comprises a positively charged n-region, a hydrophobic h-region and a c-region with the consensus sequence [LVI][ASTVI][GAS] followed by an invariant $\mathrm{C}$ residue, known as the lipobox (Babu et al., 2006). Translocation of the preprolipoprotein across the inner membrane (IM) occurs predominantly via the general secretory or Sec pathway (Driessen and Nouwen, 2008). Both the n-region and h-region are involved in interaction with SecA or other chaperones which deliver the precursor protein to the Sec-YEG transmembrane channel (Mori et al., 1997). Preprolipoprotein diacylglyceryl transferase, Lgt, transfers the diacylglyceryl group from phosphatidylglycerol to the conserved $\mathrm{C}$ residue (Sankaran and $\mathrm{Wu}, 1994$ ). This diacylglyceryl modification of preprolipoproteins is vital for substrate recognition by the dedicated lipoprotein signal peptidase LspA which cleaves the SP (Tokunaga et al., 1982; Inouye et al., 1983; Vogeley et al., 2016). In diderms, such as Neisseria, the amino group of the $\mathrm{C}$ residue is further modified with a third acyl chain through apolipoprotein $\mathrm{N}$-acyltransferase, Lnt (da Silva et al., 2017). Fully processed lipoproteins (cleaved and lipidated) that are destined to be anchored to the OM are transported by the Lol (lipoprotein OM localization) machinery (Tokuda, 2009). We previously showed that in N. meningitidis, Lol consists of the IM ABC transporter-like complex, LolFD (as opposed to LolCDE of Escherichia coli) the periplasmic LolA chaperone and the OM LolB lipoprotein receptor (da Silva et al., 2017) (Figure 1). FHbp is then surface localized by lipoprotein assembly modulator (Slam) (Hooda et al., 2016). Slam either acts as a conduit for FHbp or delivers it to another $\mathrm{OM}$ protein or to a complex such as Bam (Hooda et al., 2016) (Figure 1).

Through an accelerated approval process, both Trumemba (Pfizer) and Bexsero (GSK) were licensed by the FDA in 2014 and 2015 respectively for immunization to prevent invasive disease by meningococcal group B in the United States in individuals 10 to 25 years of age. Trumenba comprises two recombinant FHbps, one from subfamily A, the other from subfamily B, both containing the lipid moiety found in the native protein (Fletcher et al., 2004; Gandhi et al., 2016). A recombinant non-lipidated form of FHbp from subfamily B is also one of the antigens of the Bexsero vaccine (GSK) (Vernikos and Medini, 2014) licensed for infants from 2 months of age in Europe in 2013 and, like Trumenba, now licensed globally (Basta and Christensen, 2016).
A concern however for FHbp-based vaccines is the variation in expression level of FHbp between strains by over 15-fold and a threshold level of expression of at least 757 molecules of FHbp per cell is required for killing by FHbp-specific antibodies, meaning that isolates with low FHbp surface decoration fail to be effectively targeted (Jiang et al., 2010; Zlotnick et al., 2015; Biagini et al., 2016). The regulation of FHbp expression is influenced by external factors; at the transcriptional level by both iron and oxygen availability and at the translational level, by temperature (Oriente et al., 2010; Sanders et al., 2012; Loh et al., 2016). Our work has focused on bacterial-cell-intrinsic molecular factors, which govern the processing and localization of FHbp to the surface (da Silva et al., 2017) (Figure 1) which is key for target recognition following immunization with FHbpbased vaccines.

One of the studies correlating surface exposure of FHbp and susceptibility to killing by anti-FHbp antibodies was conducted by Newcombe et al. (2014) [27]. While strain L91543 displayed very little FHbp at the surface and demonstrated lack of killing by anti-FHbp sera, strain MC58, a well-characterized reference strain, demonstrated strong surface expression of FHbp and efficient killing by anti-FHbp sera (Newcombe et al., 2014). We further observed a difference in size in FHbp between these two strains, despite their high amino acid (AA) sequence identity across the same length open reading frame (Figure 2A) (Karlyshev et al., 2015). The FHbp of strain MC58 is of the expected size; $27 \mathrm{kDa}$ (Schneider et al., 2009) and is known to be tri-palmitoylated at the $\mathrm{C}$ residue (Mascioni et al., 2010) whereas the FHbp of L91543 is about $3 \mathrm{kDa}$ larger (Figure 2A) (Karlyshev et al., 2015). We identified two nonsynonymous single nucleotide polymorphisms (SNPs) in the h-region of the FHbp SP of L91543 that we hypothesized affects translocation and processing and ultimately localization to the cell surface (Karlyshev et al., 2015). In this study, by restoring these SNPs we provide strong evidence to support our hypothesis.

Surprisingly we found that the majority of invasive isolates circulating in the United Kingdom carry these SP SNPs and exclusively express the preprolipoprotein, which is partially retained in the cytoplasm due to reduced binding to SecA. Of the precursor protein that is translocated to the IM, some is retained in the periplasm and some exported to the surface. Our data suggest translocation to the OM is facilitated by Lnt and surface display is conducted by Slam following escape from processing by Lgt and LspA. Our findings thus suggest novel roles for Lnt as a chaperone facilitating periplasmic transport and for Slam in localizing non-lipidated FHbp to the cell surface. We show that the reduced surface display of precursor FHbp, due to cytoplasmic retention, correlated with reduced killing by FHbp-specific antibodies. Knowledge of the SP sequence of isolates in addition to the promoter and upstream regulatory sequence of FHbp (Cayrou et al., 2018) will enable improved prediction of surface abundance and thus coverage by Trumenba. New algorithms can now be deployed to predict whether other preprolipoproteins are processed to become mature lipoproteins or remain as precursors. This manuscript has been released as a Pre-Print at BioRxiv (da Silva et al., 2019). 


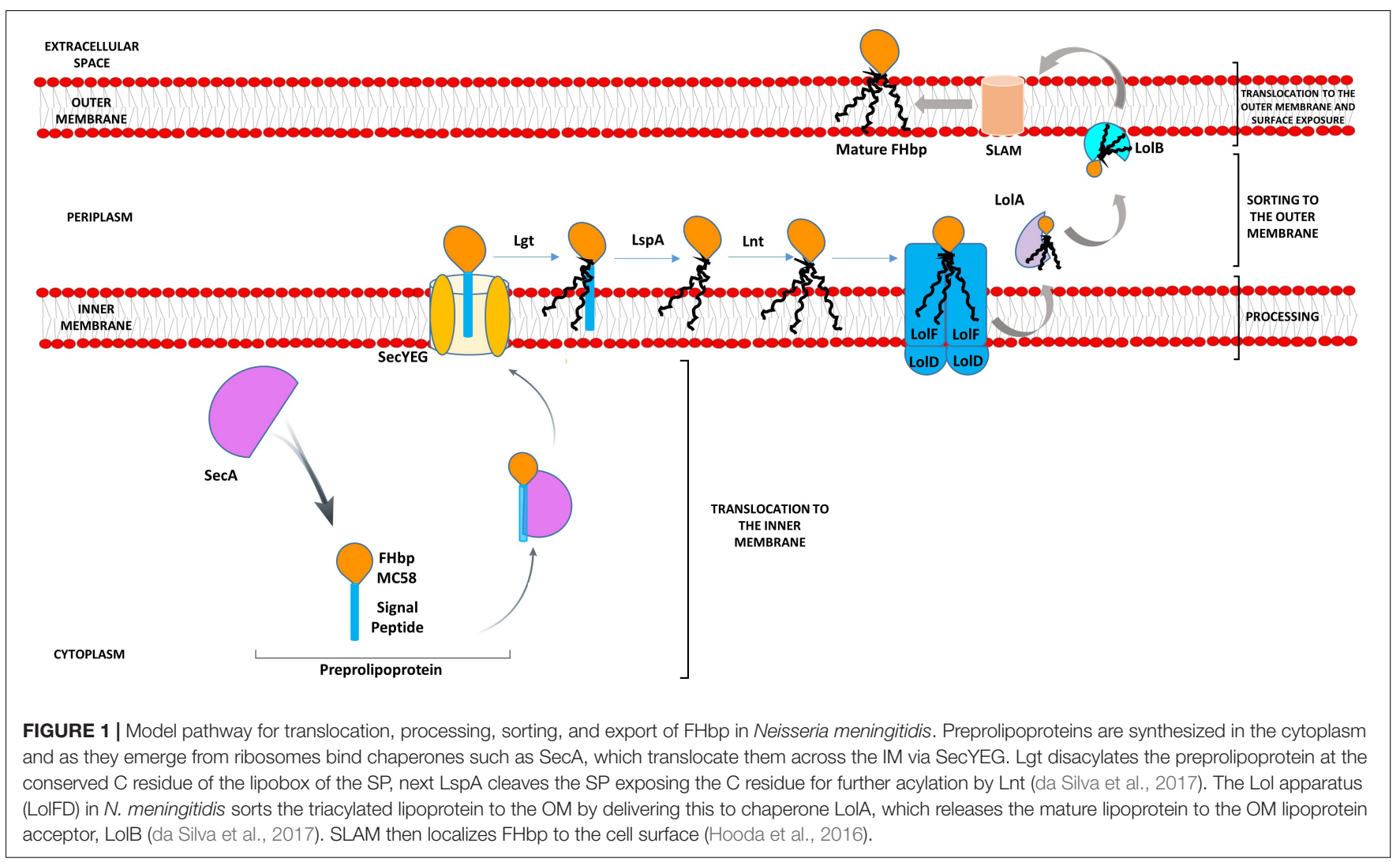

\section{MATERIALS AND METHODS}

\section{Bacterial Strains and Culture Conditions}

Escherichia coli strain JM109 single use competent cells were purchased from Promega and used for transformations in E. coli. BTH101 (Euromedex) was used as a reporter strain for BACTH assays.

The two reference (control) strains of N. meningitidis used in this study are MC58, serogroup B:15:P1.7,16, ST-74; ET-5 purchased from LGC Standards and L91543 serogroup C:2aP1.2, ST-11; ET-37 kindly provided by Professor McFadden (University of Surrey) (Table 1). Strain H44/76, serogroup B:P1.7,16:F3-3: ST-32 was gifted by Rob Read and all other group B isolates were given by Christopher Bayliss with approval from Ray Borrow (Public Health England) (Table 2) and are listed in the Meningococcus Genome Library database (MRF collection). These isolates were obtained from patients in England, Wales, Northern Ireland and Scotland from 2009 to 2017.

Escherichia coli strains were grown at $37^{\circ} \mathrm{C}$ in Lysogeny broth (LB) with shaking at $200 \mathrm{rpm}$ or on agar (Merck). All meningococcal strains were grown on GC agar plates or GC broth (Difco) containing Kellogg's glucose and iron supplements (Kellogg et al., 1963) in a moist atmosphere containing 5\% $\mathrm{CO}_{2}$ at 37 or $30^{\circ} \mathrm{C}$ for transformation experiments and with shaking at $220 \mathrm{rpm}$ for broth cultures.

Antibiotics were purchased from Sigma and added at the following concentrations: kanamycin, 30 and $60 \mu \mathrm{g} / \mathrm{ml}$, erythromycin, 300 and $0.3 \mu \mathrm{g} / \mathrm{ml}$ for E. coli and N. meningitidis respectively; $100 \mu \mathrm{g} / \mathrm{ml}$ ampicillin for E. coli; and $30 \mu \mathrm{g} / \mathrm{ml}$ nalidixic acid for E. coli BTH101.

\section{Antibodies}

Mouse anti-FHbp-Mabs, JAR4 and JAR5, were obtained from NIBSC. JAR4 is IgG2a and JAR5 is IgG2b; both were obtained from mice immunized with recombinant FHbp derived from MC58 (Welsch et al., 2004). Mouse anti-FHbp polyclonal antibody was kindly provided by Christoph Tang and rabbit antiRecA IgG was purchased from Abcam. Factor $\mathrm{H}$ protein was purchased from Bio-Rad and the mouse monoclonal IgG1 Factor H Antibody (OX24) conjugated with PE purchased from Santa Cruz Biotechnology. Secondary antibodies included donkey antirabbit HRP-linked IgG, sheep anti-mouse HRP-linked IgG (GE Healthcare, United Kingdom) for Western immunoblotting and rat anti-mouse IgG $\mathrm{H}+\mathrm{L}$ conjugated with FITC for FACs analysis (Thermo Fisher Scientific).

\section{SDS-PAGE, Western Immunoblotting, and Immuno-Dot Blotting}

Whole cell lysates (WC) were prepared from broth or plate cultures resuspended in PBS fractionated by 12 or $16 \%(\mathrm{w} / \mathrm{v})$ SDS-PAGE and immunoblotted as described previously (da Silva et al., 2017). For immunoblotting, membranes were incubated with JAR4 (diluted 1:1000 from $1 \mathrm{mg} / \mathrm{ml}$ stocks) or rabbit anti-RecA antibody diluted (1:1000) for 1 day, washed then incubated with either sheep anti-mouse or donkey anti-rabbit HRP-linked secondary antibody. Protein 


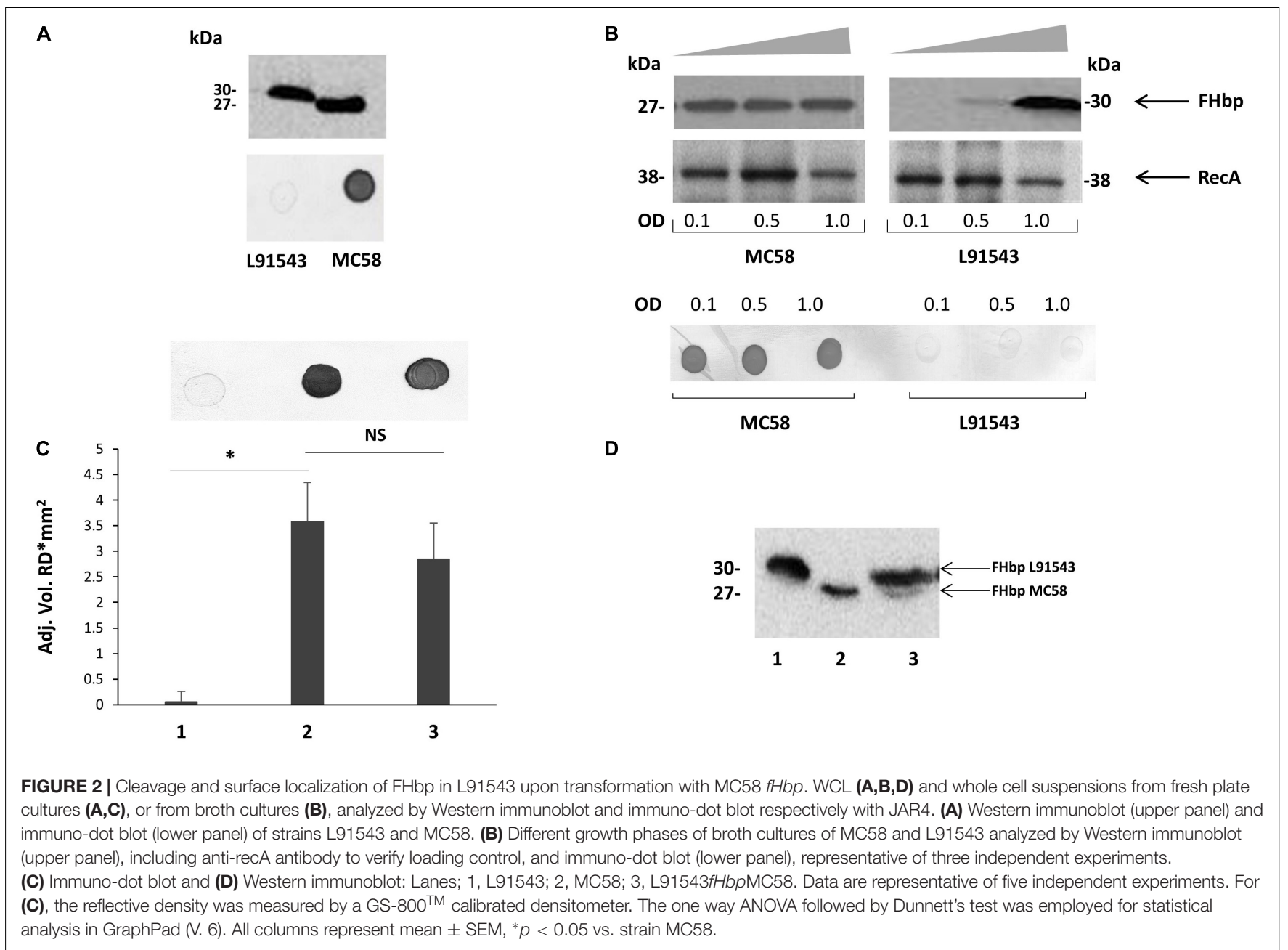

bands were detected by enhanced chemiluminescence (GE Healthcare, United Kingdom) or 3,3',5,5'-tetramethylbenzidine (TMB) (Sigma). Band intensity was quantified using a GS$800^{\mathrm{TM}}$ calibrated densitometer (Bio-Rad) or ImageJ 1.x (Schneider et al., 2012) calibrated to perform Optimal Density (OD) based on a pallet of colors in grayscale. Immuno-dot blots were performed as described previously (da Silva et al., 2017).

\section{Palmitate Labeling of Lipidated Proteins}

Bacterial cultures were grown in supplemented GC broth then after an initial doubling period, alkyne-labeled palmitic acid (Cayman Chemical) was added to a final concentration of $45 \mu \mathrm{M}$. Bacteria were incubated for at least four more hours at $37^{\circ} \mathrm{C}$.

\section{Immuno-Precipitation of FHbp From Precipitated Supernatant}

Samples were immuno-precipitated with Protein G Mag Sepharose (GE Healthcare Life Sciences) and Mab JAR4. Following incubation of $100 \mu \mathrm{l}$ of precipitated sample with $5 \mu \mathrm{g}$ of JAR4 overnight at $4^{\circ} \mathrm{C}$, samples were incubated for $1 \mathrm{~h}$ with $100 \mu \mathrm{l}$ of magnetic bead slurry. Using a magnetic particle concentrator (MPC), beads were washed twice with PBS and $\mathrm{FHbp}$ recovered following the addition of $100 \mu \mathrm{l} 0.1 \mathrm{M}$ glycine- $\mathrm{HCl}$ ( $\mathrm{pH} 2.5$ to 3.1). Buffer exchange from glycine$\mathrm{HCl}$ buffer to click reaction buffer (100 mM Na-Phosphate Buffer, pH 7) was performed with Slide-A-Lyzer Dialysis cassettes (Sigma).

\section{Click Chemistry}

The Click Chemistry labeling system "CuAAC Biomolecule Reaction Buffer Kit (THPTA based)" (Jena Bioscience) was used following manufacturer's instructions (Ostberg et al., 2013). FHbp was coupled to biotin azide (Stratech) then samples fractionated on 10-20\% (w/v) SDS-PAGE gels (Novex). Western immunoblotting was performed by incubating the membrane with Streptavidin HRP-linked protein in PBS-BSA buffer and developing with TMB (Sigma).

\section{Harvesting Cellular Compartments of N. meningitidis}

Periplasmic extracts were prepared using a method previously described (Ames et al., 1984). Cells from over-night GC plate cultures were suspended to $A_{600} 1.0$ in $500 \mu$ l buffer $(50 \mathrm{mM}$ 
TABLE 1 | Frequency of different FHbp SP classes in capsular group B isolates in the MRF Meningococcus Genome Library database.

\begin{tabular}{|c|c|c|c|}
\hline SP class & SP sequence & No. of isolates (\%) $n=1895$ & Isolates used in this study \\
\hline 1 (NO SNPS) & MTRSKPVN R TAFCCLSLITIALI LTAC & $171(9)$ & MC58 and 1-4 \\
\hline \multirow[t]{2}{*}{2 (SNP1 and SNP2) } & MTRSKPVN R TAFCCFESLTÁAU LTAC & $435(23)$ & L91543 and 10-13 \\
\hline & SNP1 SNP2 & & \\
\hline \multirow[t]{2}{*}{3 (SNP2) } & MTRSKPVN R TAFCCLSLTÁALI LTAC & $910(48)$ & $5-9$ \\
\hline & SNP2 & & \\
\hline \multirow[t]{2}{*}{4 (SNP2 and SNP3) } & MTRSKPVN R TIIFCCLSLTAALI LTAC & $323(17)$ & $14-18$ \\
\hline & $\begin{array}{ll}\text { SNP3 } & \text { SNP2 }\end{array}$ & & \\
\hline Other SNPs & & $56(3)$ & \\
\hline
\end{tabular}

The n-region is shown in brown, h-region in green and lipobox in red, corresponding to the display generated by the DOLOP lipoprotein prediction program (https: //www.mrc-Imb.cam.ac.uk/genomes/dolop/analysis.shtml) (Babu et al., 2006).

\begin{tabular}{lcc}
\hline TABLE 2 | MenB invasive isolates used in this study. & \\
\hline Class & Isolate number & Isolate, full name \\
\hline 1 & - & MC58 \\
& 1 & H44/76 \\
& 2 & M10_240684 \\
& 3 & M10_240701 \\
4 & M02_241729 \\
& 5 & M10_240579 \\
3 & 6 & M13_240525 \\
& 7 & M04_241215 \\
& 8 & M11_241066 \\
& 9 & M13_240614 \\
& - & L91543 \\
10 & M10_240750 \\
11 & M13_240675 \\
12 & M11_240236 \\
13 & M12_240006 \\
14 & M11_241033 \\
15 & M14_240367 \\
16 & M02_240210 \\
17 & M11_240077 \\
18 & M13_240486 \\
\hline & &
\end{tabular}

The SP class is indicated for each isolate.

Tris- $\mathrm{HCl} \mathrm{pH}$ 8.0) and pelleted at $3,500 \times g$ for $2 \mathrm{~min}$. The pellet was resuspended in $200 \mu \mathrm{l}$ of the same buffer and $20 \mu \mathrm{l}$ of chloroform was added. After brief vortexing, tubes were incubated for $15 \mathrm{~min}$ at room temperature. After centrifugation at $6,500 \times g$ for $2 \mathrm{~min}$, the upper portion of the supernatant, containing the periplasmic proteins, was carefully aspirated and placed in a second tube. The pellet containing the remaining IM, OM, and cytoplasmic proteins was partitioned following approaches adapted from two different research groups (Clark et al., 1987; Rahman et al., 2000). The pellet was re-suspended in $500 \mu \mathrm{l}$ buffer $[50 \mathrm{mM}$ Tris$\mathrm{HCl} \mathrm{pH} \mathrm{8.0,20 \%} \mathrm{(w/v)} \mathrm{sucrose]} \mathrm{containing} 1 \mathrm{mg} / \mathrm{ml}$ lysozyme and incubated for $30 \mathrm{~min}$ at $4^{\circ} \mathrm{C}$. After two cycles of freezethawing, the cells were subjected to sonication ( 2 bursts of $30 \mathrm{~s}$ ). Cellular debris were removed by centrifugation at $9,500 \times g$ for $10 \mathrm{~min}$. Ultra-centrifugation at $100,000 \times g$ for $60 \mathrm{~min}$ enabled partitioning of the membranes in the pellet from cytoplasmic proteins in the supernatant. The IM was selectively solubilized by treatment with $200 \mu \mathrm{l}$ of $1 \%$ (w/v) sodium lauroyl sarcosinate in $10 \mathrm{mM}$ HEPES ( $N$-2-hydroxyethylpiperazine $N^{\prime}$ 2-ethanesulfonic acid) $\mathrm{pH} 7.4$ buffer. After centrifugation at $100,000 \times g$ for $1 \mathrm{~h}$ the supernatant containing solubilized IM proteins was separated from the pellet containing OM proteins. The pellet was washed with ethanol and resuspended in $200 \mu \mathrm{l} \mathrm{PBS}$.

\section{Molecular Methods for DNA Manipulations}

Genomic DNA was extracted from N. meningitidis using the Gentra Puregene Yeast/Bact Kit (Qiagen) and plasmid DNA was extracted from E. coli using the QiaPrep Spin kit (Qiagen). DNA samples were analyzed by agarose gel electrophoresis and visualized by staining with SYBR Safe (Invitrogen). Restriction enzymes (NEB), T4 DNA ligase (Promega), and Antarctic Phosphatase (NEB) were used according to the manufacturer's recommendations. PCRs were performed using Q5 polymerase kit (NEB) in a Perkin- MJ Research PTC-200 Peltier Thermal Cycler or C1000 Touch ${ }^{\text {TM }}$ Thermal Cycler (Bio-Rad). Primers were purchased from Sigma and their sequences listed in Table 3. PCR products and restriction digested DNA were purified using the PCR Mini Elute kit (Qiagen). E. coli was transformed by heat shock (Froger and Hall, 2007).

\section{Genome Sequencing}

The N. meningitidis L91543 genome sequence was completed using a hybrid approach employing a combination of short and long reads assemblies produced by IonTorrent (Karlyshev et al., 2015) and PacBio technologies. PacBio sequencing was conducted at TGAC (The Genome Analysis Centre, Norwich, United Kingdom) using an RSII sequencing machine with P6/C4 sequencing chemistry and a single SMRT cell. When analyzing the PacBio assembly, long redundant regions were detected at the ends of the sequence. CLC Genomics Workbench software and high quality reads generated by IonTorrent PGM were used for elimination of artifactual redundancies and for circularization, as well as for final 
TABLE 3 | PCR primer pairs used in this study.

PCR, sequencing and RT-PCR

Primer name

Primer sequence

fHbp cloning (restriction sites underlined)

Pacl-fHbp-for

Pmel-fHbp-rev

5'-GCGCAATTAATTAATTGCTTCTTGACCTGCC-3'

pGCC4 sequencing primers

pGCC4-fwd

5'-ACCTGTTTAAACAATGGTTATTGCTTGGCG-3'

pGCC4-rev

5'-AGACATCCGCCAAACCATCC-3'

RT-PCR primers

fHbp-for

fHbp-rev

5'-TGCTTCCGGCTGTTGTGTGG-3'

recA-for

recA-rev

Bacterial two-hybrid (restriction sites underlined)

BamHIfwd_MC58FHbp

EcoRIrev_MC58FHbp

BamHlfwd_L91543FHbp

EcoRIrev_L91543FHbp

Pstlfwd_SecA

Smalreverse_SecA

Site-directed mutagenesis (incorporated mutations italicized)

SP1_Fwd

SP1_Rev

SP2_Fwd

SP2_Rev

5'-GTTTCGCAACCATCTTCCCG-3'

5'-GACTTTATCCGTCAAATCGA-3'

5'-GAAGAGGTATTGGCAACGA-3'

5' - CGGATTTGTTGATGATGTCG-3'

5'-GCGAGGATCCATGACTAGGAGTAAACCTGTGAATC-3'

5'-GATCGAATTCTTATTGCTTGGCGGCAGGCCGATATG-3'

5'-GCGAGGATCCATGCCGTCTGAACCGTTGTTCGGACGGC-3'

5'-GATCGAATTCTTACTGCTTGGCGGCAAGACCGATATGG-3'

5'-CGATCTGCAGATGCTGACAAACATTGCCAAGAAAATC-3'

5'-CGACCCGGGTTAAGCCAGTTGCCGTGGCATTG-3'

Deletion of $f H b p$ by SOEing

HA1_FHbp_Fwd

Rev_HA1_FHbp

Kan_fwd

5'-CTTCTGCTGCCTTCTCTGACCG-3'

5'-GCAGTTCGGTTCACAGGT-3'

5'-TTCTCTGACCACTGCCCTGATTC-3'

5'-AAGCAGCAGAAGGCAGTT-3'

Kan_rev

HA2_FHbp_fwd

HA2_FHbp_rev

5'-GATAGAATTCCGAGTATGCAGCTITG-3'

5'-GATGATGGTTGCCATTGTGAAAATGCCGTCC-3'

5'-TICACAATGGCAACCATCATCGATG-3'

5'-AAACCTTTCAGACGGCATGTAATGCTCTGCC-3'

5'-ATGCCGTCTGAAAGGTTIACTCCTAGTCATACG-3'

5'-CTTAGGATCCCCACGGCGCATACAAATTC-3'

Deletion of slam by SOEing (kan sequence in bold, HA sequence underlined, overlapping sequence italicized)

HA1_0313_Fwd

5'-GATAGAATTCAGGCGCAGTTACCTACTTG-3'

HA1_0313_rev

5'-GATGATGGTTGTATCAATCGGCGGATTGTATC-3'

0313_Kan_fwd

5'-GATACAATCCGCCGATTGATACAACCATCATCGATG-3'

0313_Kan_rev

5'-AACAGCAATTCAGACGGCATGTAATGCTCTGCC- $3^{\prime}$

HA2_0313_fwd

5'-ATGCCGTCTGAATTGCTGTTCCTITCGGAGG-3'

HA2_0313_rev

5'-CTTAGGATCCGAACGGCTTATGGCTTGGGAC-3'

Disruption of Lnt

Lnt_fwd

5'-GGCAGGAGATATGCGCTAAG-3'

Lnt_rev

5'-GTACTGGTCGCCCACAACCT-3'

verification of the sequence and correction of any errors produced by PacBio.

\section{BLAST Analysis}

Whole genome sequence (WGS) data from capsular group B isolates with complete FHbp profiles in the Meningitis Research Foundation Meningococcus Genome Library database (containing WGS data from all United Kingdom disease isolates from 2010 to 2017) was interrogated using the BLAST tool implemented within the
PubMLST.org/neisseria database using default settings (last analyzed September, 2018).

\section{Construction of Strain L91543fHbpMC58}

The region incorporating the promoter and open reading frame of $f H b p$ was PCR-amplified from MC58 genomic DNA with primers PacI-fHbp-for and PmeI-fHbp-rev (Table 3) digested with PacI and PmeI sites and cloned into the PacI, PmeI sites of Neisseria complementation vector, pGCC4 (Addgene) (Mehr and Seifert, 1998). The resulting plasmid, 
pGCC4fHbpMC58 was verified by DNA sequencing with pGCC4 primers (Table 3) and the plasmid used to transform N. meningitidis strain L91543 as previously described (Zhang et al., 2010) with selection on erythromycin. A transformant was verified by PCR with subsequent DNA sequencing and designated L91543fHbpMC58. The same approach was used to create plasmid pGCC4fHbpL91543 with the L91543 version of $f H b p$, which was used for site-directed mutagenesis.

\section{Construction of Strain L91543 $\Delta \mathrm{fHbp}$}

Gene Splicing by Overlap Extension (gene SOEing) was used to create a fusion PCR product to replace fHbp in strain L91543 with the kanamycin resistance gene ( $k a n)$ from EZ:Tn5 < KAN$2>$ insertion kit (Epicentre) following the approach described by Horton (Horton, 1995). In the first round of PCRs, homology arms (HA1 and HA2) of approximately 600 bp flanking $f H b p$ were amplified from genomic DNA of L91543 using primers, HA1_FHbp_Fwd and Rev_HA1_FHbp and HA2_FHbp_fwd and HA2_FHbp_rev, and the kan gene was amplified using primers Kan_fwd and Kan_Rev. As shown in Table 3, in bold are the regions that bind to $k a n$, underlined are the regions that bind to the HA of interest and in italics are the regions that over-lap. The HA1 and kan PCR products obtained were genecleaned then used as template for the second round of PCR with primers HA1_FHbp_Fwd and Kan_Rev and the annealed product generated cleaned and used as template along with the HA2 PCR product for a third round of PCR with primers HA1_FHbp_Fwd and HA2_FHbp_rev. The final PCR product generated containing HA1-kan-HA2 was gene cleaned and sequenced for confirmation. The verified construct was used to transform strain L91543 with selection on kanamycin. Deletion mutants were confirmed by PCR and DNA sequencing and designated L91543 $\Delta f H b p$.

\section{Disruption of Slam}

In the same manner as that described above, the gene encoding Slam (NMB0313) was replaced with kan. For the first round of PCRs, primers HA1_0313_Fwd and HA1_0313_rev and HA2_0313_fwd and HA2_0313_rev were used to amplify approximately $600 \mathrm{bp}$ of sequence flanking slam from genomic DNA of MC58 and kan was amplified with primers 0313_Kan_fwd and 0313_Kan_rev (Table 3). The appropriate primer pairs were used in second and third round PCR and the construct confirmed by sequencing then used to transform MC58, L91543 and isolates 6 and 18 with selection on kanamycin. Insertion mutants were confirmed by PCR and DNA sequencing and designated MC58Slam, L91543Slam, Isolate6Slam, and Isolate18Slam.

\section{Disruption of Lnt}

Primers, Lnt_fwd and Lnt_rev (Table 3), annealing approximately 600 bp upstream and downstream respectively of $\ln t$ in strain MC58Lnt, were used to amplify $\ln t$ that had been disrupted by the insertion of Tn $5<\mathrm{KAN}-2>$ (Epicentre) (da Silva et al., 2017). The PCR product generated was used to transform L91543 and isolates 6 and 8 with selection on kanamycin. Deletion mutants were confirmed by PCR and
DNA sequencing and designated L91543Lnt, Isolate6Lnt, and Isolate18Lnt.

\section{Site-Directed Mutagenesis}

Site-directed mutagenesis of the fHbp SP in pGCC4fHbpL91543 to repair SNP1 and SNP2 individually was performed using the Q5 ${ }^{\circledR}$ Site-Directed Mutagenesis Kit (NEB) according to the manufacturer's recommendations. SNP1 was corrected to create plasmid pGCC4LfHbpSNP1 and SNP2 was corrected to create plasmid pGCC4LfHbpSNP2. Seven ng of pGCC4fHbpL91543 and $0.5 \mu \mathrm{M}$ of each pair of primers (Sigma) were used for amplification and incorporation of the desired mutation. Primers SP1_Fwd and SP1_Rev, and SP2_Fwd and SP2 _Rev (Table 3) were used to incorporate SNP1 and SNP2 respectively. PCR was performed in a C1000 Touch $^{\mathrm{TM}}$ Thermal Cycler (Bio-Rad) with the following thermo-cycling conditions; $98^{\circ} \mathrm{C}$ for $30 \mathrm{~s}$ followed by 25 cycles of $98^{\circ} \mathrm{C}$ for $10 \mathrm{~s}$ (denaturation), $68^{\circ} \mathrm{C}$ for $15 \mathrm{~s}$ (annealing), $72^{\circ} \mathrm{C}$ for $3 \mathrm{~min}$ and $25 \mathrm{~s}$ (extension) and $72^{\circ} \mathrm{C}$ for 2 min (final extension). One $\mu \mathrm{l}$ of the PCR product was kinase-, ligase-, and $D p n I$ - treated (KLD treatment) and incubated for $5 \mathrm{~min}$ at room temperature. Five $\mu \mathrm{l}$ of the KLD mix was then used to transform competent cells. After plating on LB agar with kanamycin and incubating overnight, several colonies were isolated, grown individually in LB broth with kanamycin and plasmid DNA extracted and sequenced with pGCC4 primers (Table 3) for verification. To repair both SNPs, the PacI-PmeI fragment of pGCC4fHbpL91543 was commercially synthesized (Life Technologies) with the two SNPs repaired to resemble the fHbp SP of MC58 and the DNA cloned into the PacI-PmeI sites of pGCC4 to create pGCC4LfHbpSNP1 +2 . The construct was verified by sequencing with pGCC4 primers (Table 3 ).

Strain L91543 $\Delta f H b p$ was transformed with pGCC4 $f H b p \mathrm{~L} 91543$ to create L91543 $\Delta f H b p \mathrm{~L} f H b$ with no SNP corrections as a negative control. The same strain was transformed with pGCC4L $f H b p S N P 1$ to generate recombinant strain L91543 $\Delta f H b p+\mathrm{L} f H b p S N P 1$, with pGCC4L $f H b p S N P 2$ to generate strain L91543 $\Delta f H b p+\mathrm{L} f H b p S N P 2$ and finally with pGCC4L $f H b p S N P 1+2$ to create strain L91543 $\Delta f H b p+$ $\mathrm{L} f H b p \mathrm{SNP} 1+2$.

\section{Bacterial Two-Hybrid Assay (BACTH)}

The protein-protein interaction of MC58 FHbp and L91543 FHbp with SecA was investigated using the Bacterial Adenylate Cyclase Two-Hybrid System Kit (Euromedex) according to manufacturer's instructions. First, fHbp from MC58 and from L91543 was PCR-amplified with the primer pair BamHIfwd_MC58FHbp and EcoRIrev_MC58FHbp and primer pair BamHIfwd_L91543FHbp and EcoRIrev_L91543FHbp respectively (Table 3 ) then the PCR products cloned separately into vector pUT18. The gene encoding SecA from MC58 was PCR-amplified with primer pair, PstIfwd_SecA and SmaIreverse_SecA (Table 3) then cloned into vector pKT25. 25-50 ng of the appropriate prey (pKT25-based construct) and the equivalent concentration of appropriate bait (pUT18-based construct) were co-transformed into $100 \mu \mathrm{l}$ of competent E. coli BTH101 cells and plated on MacConkey agar containing $0.5 \mathrm{mM}$ IPTG and appropriate antibiotics. Bacteria expressing 
interacting hybrid proteins formed pink/purple colonies while cells expressing non-interacting proteins remained white/light pink. As a positive control, a co-transformant containing commercial pKT25-zip and pUT18-zip constructs was used. Cotransformants containing empty vector pKT25 and/or pUT18 were used as negative controls. Pink colonies were isolated and grown in LB broth and plasmid DNA extracted for verification by PCR and sequencing.

\section{$\beta$-Galactosidase Assay}

Following the approach previously described (Chambonnier et al., 2016), to measure the level of protein-protein interaction between FHbp from MC58 or L91543 with SecA, LB broth cultures were grown to $A_{600} 0.6$ then induced for $3 \mathrm{~h}$ with $0.5 \mathrm{mM}$ IPTG. Five $\mu \mathrm{l}$ of induced culture were mixed with $900 \mu \mathrm{l}$ of $\mathrm{Z}$ buffer $\left(0.06 \mathrm{M} \mathrm{Na} 2 \mathrm{HPO}_{4} 2 \mathrm{H}_{2} \mathrm{O}, 0.04 \mathrm{M} \mathrm{NaH} \mathrm{PO}_{4}\right.$, $0.01 \mathrm{M} \mathrm{KCl}, 2 \mathrm{mM} \mathrm{MgSO} 4.7 \mathrm{H}_{2} \mathrm{O}, 14.20 \mathrm{M} \beta$-mercaptoethanol $(\mathrm{pH} 7)$, before addition of $20 \mu \mathrm{l}$ of $0.1 \%(\mathrm{w} / \mathrm{v})$ SDS and $100 \mu \mathrm{l}$ of $\mathrm{CHCl}_{3}$ to permeabilize the cells. Substrate solution was prepared by solubilizing orthonitrophenyl- $\beta$-galactosidase (Sigma) in $\mathrm{Z}$ buffer without the $\beta$-mercaptoethanol to a final concentration of $4 \mathrm{mg} / \mathrm{ml}$. $40 \mu \mathrm{l}$ of substrate solution were added to $180 \mu \mathrm{l}$ of $\mathrm{Z}$ buffer and $20 \mu \mathrm{l}$ of permeabilized cells in a 96-well plate. The plate was incubated at room temperature for at least $20 \mathrm{~min}$. Readings were taken on a microplate reader at $A_{405}$ and $A_{540}$ and $\beta$-galactosidase activity calculated using the equation below and expressed in Miller units.

$\beta-$ galactosidase activity $=$

$\frac{1000 \times\left(A_{405}-A_{540}\right)}{\text { Time }(\mathrm{min}) \times \text { vol. of cells in assay }(\mathrm{ml}) \times A_{600}}$

\section{FACS Analysis of Isolates to Measure the Number of JAR4 Antibodies Bound per Cell $(A B C)$ and Quantify Surface Localization of FHbp}

FHbp surface expression was assessed using a MoFlo Astrios EQ, Cell Sorter (Beckman Coulter). The approach used was similar to that described previously (Biagini et al., 2016). Approximately $1 \times 10^{5}$ bacteria were suspended in PBS containing $1 \%(\mathrm{w} / \mathrm{v})$ bovine serum albumin (PBS-BSA) and incubated with JAR4 in a final volume of $100 \mu \mathrm{l}$ for $1 \mathrm{~h}$ at $37^{\circ} \mathrm{C}$. After two washes with PBS, JAR4 binding was detected using FITC-conjugated rat anti-mouse $\operatorname{IgG}(\mathrm{H}+\mathrm{L})$ (Thermo Fisher) at a 1:50 dilution for an incubation period of $1 \mathrm{~h}$. After the final two washes with PBS, samples were resuspended in $500 \mu$ l of PBS-BSA with $4 \%(\mathrm{w} / \mathrm{v})$ formalin. The negative control consisted of cells incubated with the secondary antibody alone. Quantum ${ }^{\mathrm{TM}}$ Simply Cellular ${ }^{\circledR}$ anti-rat (Bang Laboratories) microspheres were used to determine the number of $\mathrm{ABC}$ according to the manufacturer's instructions. Median channel values for each population of microspheres were acquired for entry into the QuickCal ${ }^{\circledR}$ spreadsheet to generate a curve that enabled the acquisition of $\mathrm{ABC}$ for each isolate.

\section{FACS Analysis of Isolates With Anti-fH Antibody to Quantify fH Binding}

Approximately $1 \times 10^{5}$ bacterial cells were suspended in PBS containing $1 \%(\mathrm{w} / \mathrm{v})$ PBS-BSA and incubated with $\mathrm{fH}$ (Bio-Rad) at a final concentration of $5 \mu \mathrm{g} / \mathrm{mL}$ for $1 \mathrm{~h}$ at room temperature. After two washes with PBS, anti-fH antibody (OX-24) conjugated to $\mathrm{PE}$ was added at a 1:50 dilution for $1 \mathrm{~h}$. After two more washes in PBS, samples were resuspended in $200 \mu \mathrm{l}$ of PBSBSA with $4 \%(\mathrm{w} / \mathrm{v})$ formalin. The negative control consisted of cells incubated with anti-fH antibody alone. The relative Mean of Fluorescence Intensity (rMFI) for total fluorescent cells was acquired for each sample and these values normalized against rMFI of cells incubated with secondary antibody alone.

\section{Susceptibility of Isolates to Killing in Serum Bactericidal Antibody Assays}

The susceptibility of the isolates to complement-mediated killing with antibodies JAR4 and JAR5 was assessed using approaches adapted from previous studies (Beernink et al., 2008, 2011; Vu et al., 2012). JAR4 and JAR5 were used in combination, in place of serum antibodies, to generate bactericidal activity (Beernink et al., 2008; Welsch et al., 2008; Malito et al., 2016). Eight $\mu \mathrm{g}$ of each antibody were used to ensure maximum killing (Beernink et al., 2008). Complement was obtained from lyophilized human sera (Sigma) reconstituted in sterile water as per manufacturer's instructions. Complement and antibodies were added to IgGdepleted human sera (Stratech) that had been heat-inactivated for $30 \mathrm{~min}$ at $56^{\circ} \mathrm{C}$. The bactericidal activity, i.e., the percentage of killing by the antibodies for each isolate was then determined from the CFU counts after $60 \mathrm{~min}$ incubation in the reaction mixture and compared with CFU counts from negative control wells at time 0 .

\section{Reverse Transcription PCR (RT-PCR)}

Following a similar approach to that we described previously (da Silva et al., 2017), RNA was extracted from $1 \mathrm{ml}$ cell suspensions of each isolate standardized to $A_{600} 0.65$ (containing approximately $2 \times 10^{8}$ cells) using the RNeasy Mini kit (Qiagen) with enzymatic lysis and Proteinase $\mathrm{K}$ digestion. On-column DNA digestion was performed using the RNase Free DNase kit (Qiagen). RNA quality was assessed for genomic contamination and integrity using a NanoDrop Lite (Thermo Fisher Scientific) and running $1 \mu \mathrm{l}$ of sample on a $1 \%$ agarose gel.

One $\mu \mathrm{g}$ of cDNA was synthesized using the QuantiTect reverse transcription kit (Qiagen) with the initial genomic wipeout step included. RT-PCR was performed in a $50 \mu \mathrm{l}$ reaction mixture with One Taq 2x master mix (NEB), 20 ng of cDNA and $0.2 \mu \mathrm{M}$ of each primer (Sigma). For amplification of cDNA of $f H b p, f H b p$-for, and $f H b p$-rev primers were used and for amplification of $r e c A$, $r e c A$-for, and $r e c A$-rev primers were used (Table 3). To qualitatively check for different levels of expression, PCR was performed in a C1000 Touch $^{\mathrm{TM}}$ Thermal Cycler (Bio$\mathrm{Rad}$ ) with the following thermo-cycling conditions; $94^{\circ} \mathrm{C}$ for $30 \mathrm{~s}$ followed by 35 cycles of $94^{\circ} \mathrm{C}$ for $15 \mathrm{~s}$ (denaturation), $56^{\circ} \mathrm{C}$ for $15 \mathrm{~s}$ (annealing) and $68^{\circ} \mathrm{C}$ for $20 \mathrm{~s}$ (extension). PCR products were visualized on a $1 \%$ agarose gel. 


\section{Statistical Analysis}

Data are shown as mean \pm SEM. Multiple comparisons between groups were performed by one way ANOVA followed by Dunnett's test. Unpaired $t$-tests with Welsh's corrections were used for comparisons between two groups. A value of $p \leq 0.05$ was considered statistically significant. Post hoc tests were run if $\mathrm{F}$ achieved $p<0.05$ and there was no significant variance. All statistical analyses were performed in GraphPad Prism version 6.00 for Windows. No statistical methods were used to predetermine sample size. The experiments were not randomized other than for selection of clinical isolates used in this study. The investigators were not blinded to allocation during experiments and outcome assessment.

\section{RESULTS}

\section{SP SNPs in FHbp Prevent Cleavage but Not Surface Localization in Strain L91543}

Neisseria meningitidis strain MC58 was originally isolated from a patient in the United Kingdom with meningococcal meningitis (McGuinness et al., 1991) and is a fully-sequenced, commonly used laboratory reference strain (Tettelin et al., 2000). Strain L91543 was isolated from the cerebrospinal fluid of a 14-year old with meningococcal meningitis in the United Kingdom (Oliver et al., 2002). The FHbp-specific monoclonal antibody, JAR4, used throughout this study was sourced from the National Institute for Biological Standards and Controls (NIBSC). JAR4 binds to an N-terminal epitope including the AA residues DHK at positions 25 to 27 (Beernink and Granoff, 2009) (Supplementary Table S1) and was generated following immunization of mice with MC58 FHbp (Welsch et al., 2004). The FHbps of both MC58 and L91543 are classified as subfamily B (Fletcher et al., 2004; Beernink and Granoff, 2009) (variant 1) (Masignani et al., 2003) and, as we previously reported, they share nucleotide and AA identities of 95 and 93\% respectively (Karlyshev et al., 2015).

We investigated differences in the surface localization of FHbp between these strains at different growth phases in a whole cell immuno-dot blot assay. A consistent strong level of surface expression of FHbp was shown for MC58 during growth in contrast to L91543, which demonstrated a uniform low level of surface localization (Figures 2A,B). Western immunoblot analysis of whole cell lysates showed a consistent high level of total FHbp expression for MC58, whereas the presence of FHbp was minimal in mid-log phase and strong only at stationary phase for L91543 cultures (Figures 2A,B). Thus for L91543, the demonstration of strong total FHbp level at stationary phase yet poor surface expression implies that FHbp is retained within the cells of this strain and fails to either sort to the OM and/or become surface-exposed, unlike MC58.

We previously reported that the FHbp of $\mathrm{L} 91543$ appears to be $3 \mathrm{kDa}$ larger than that of MC58 upon fractionation of whole cell lysates as shown in Figure 2A, this additional mass corresponding to the size of the 26 AA SP (Karlyshev et al., 2015).
To investigate any defects in LspA, which should cleave this SP, or aberrations in other proteins involved in the sequential pathway for FHbp processing and export (da Silva et al., 2017) (Figure 1), the genome of L91543 was fully sequenced (GenBank accession number CPO16684) and BLAST analysis performed using relevant query sequences from MC58. LspA is $100 \%$ identical between the two strains and all other proteins share $>97 \%$ identity with no evidence of frameshifts or premature stop codons, whereas FHbp exhibits only 93\% AA identity between these two strains (Supplementary Table S2). Notably the SP region of FHbp exhibits even greater divergence due to the presence of 2 SNPs in L91543; leucine (L) substituted by phenylalanine (F) at position 15 (SNP1) and threonine $(\mathrm{T})$ substituted by alanine (A) at position 19 (SNP2) (Table 1).

To test our hypothesis and determine whether differences in the FHbp sequence are solely responsible for the failure to cleave and export FHbp in strain L91543, the fHbp gene including its upstream promoter and ribosomal binding site from MC58 was inserted into the chromosome of strain L91543. This region was first cloned into the Neisseria complementation vector, pGCC4, which allows integration of DNA in the intergenic region between aspC and $l c t P$ without causing polar affects (Mehr and Seifert, 1998; da Silva et al., 2017). Plasmid pGCC4fHbpMC58 was transformed into strain L91543 and JAR4 was used to assess FHbp expression. Immuno-dot blot of whole cells showed that the recombinant strain, L91543fHbpMC58, acquired the ability to express FHbp at the cell surface (Figure 2C). Western immunoblot analysis of L91543fHbpMC58 revealed two bands: a lower band with a mobility equivalent to the cleaved FHbp of MC58 and a higher molecular weight band corresponding to the expected mobility for the native, uncleaved protein (Figure 2D).

To investigate the specific and combined contribution of the two individual SNPs in L91543 SP on cleavage and export, first $f H b p$ was deleted in $\mathrm{L} 91543$ (L91543 $\Delta f H b p$ ) then complemented with wild type (L91543 $\Delta f H b p+\mathrm{L} f H b p$ ) or versions with site-directed alteration in the SP $(\mathrm{L} 91543 \Delta f H b p+\mathrm{L} f H b p S N P 1, \mathrm{~L} 91543 \Delta f H b p+\mathrm{L} f H b p S N P 2$, and L91543 $\Delta f H b p+\mathrm{L} f H b p S N P 1+2)$. Western immunoblot analysis confirmed complete loss of FHbp expression in L91543 $\Delta f H b p$ (Supplementary Figure S1). L91543 $\Delta f H b p+$ LfHbpSNP1 and L91543 $\Delta f H b p+\mathrm{L} f H b p S N P 2$ showed moderate levels of surface expression and L91543 $\Delta f H b p+\mathrm{L} f H b p \mathrm{SNP} 1+2$ showed a level of surface display comparable to MC58 (Figure 3). Interestingly Western immunoblot analysis showed faster mobility, indicative of SP cleavage, for only strain L91543 $f$ Hbp + LfHbpSNP1 +2 , which had both SNPs corrected but not for either recombinant strain with individual SNPs corrected (Figure 3). Our results suggest that both AA substitutions impact on the FHbp translocationprocessing pathway at some point up to, or at, the SP cleavage step and surprisingly that repair of either SNP individually can permit partial localization of FHbp to the cell surface without the need for prior SP cleavage. This was not expected for this sequential canonical lipoprotein processing pathway. 


\section{FHbp With SP SNPs Shows Reduced Binding to SecA}

Most proteins destined for the $\mathrm{OM}$ are first targeted to the IM translocase, Sec-YEG, by the chaperone ATPase motor, SecA. SecA recognizes SPs by having a high affinity for their hydrophobic binding grooves and by electrostatically trapping their positively charged $\mathrm{n}$-region through acidic residues (Gelis et al., 2007; Gouridis et al., 2009). We investigated the binding of L91543 FHbp (carrying SNP1 and SNP2) to meningococcal SecA and compared this to MC58 FHbp, which is known to undergo cleavage and processing (Masignani et al., 2003; da Silva et al., 2017). The bacterial two-hybrid approach was taken which relies on induced expression of the two heterologous proteins using E. coli as the host organism. The cytoplasmic readout for binding affinity in this heterologous expression system is expected to be similar to that in the meningococcus, prior to translocation to the IM. Our results showed a significant 1.9-fold lower binding of SecA to L91543 FHbp as compared to MC58 FHbp (Figure 4).

\section{Clinical Isolates With FHbp SP SNPs Show SP Retention Yet Surface Localization}

We next investigated the prevalence of FHbp containing SP SNPs (that differ from the MC58 sequence) in the genome sequences of all 1,895 invasive group B United Kingdom isolates in the Meningitis Research Foundation (MRF) Meningococcus Genome Library database collected between 2009 and 2017. Unexpectedly we found that only $9 \%$ of isolates have a SP identical to that of MC58 (referred to as class 1) with the remaining isolates carrying either (i) both SNP1 and SNP2, like L91543 (class 2, 23\%); (ii) SNP2 alone (class 3, 48\%); (iii) SNP2 and a third SNP (SNP3) (class 4, 17\%), or (iv) other SNPs (3\%) (Table 1). All SNPs are located exclusively in the h-region 13 AA in length, and common to classes 2, 3, and 4 is SNP2 (i.e., T substituted by A at position 19).

To test if our findings for recombinant strains of L91543 apply to these MRF isolates with non-class $1 \mathrm{FHbp}$, a selection of isolates from each class were randomly selected (Table 2). Strain H44/76 was chosen as an additional positive control strain since its FHbp including SP is identical to that of MC58. FHbps of all 20 isolates belong to subfamily $\mathrm{B} /$ variant 1 (the most prevalent group) and possess the JAR4 epitope (Beernink and Granoff, 2009) (Table 2 and Supplementary Table S1).

FHbp from all isolates with class 1 SP demonstrated a similar size by SDS-PAGE to that of MC58 whilst all nonclass 1 FHbp proteins had the slower mobility observed for L91543 (Figure 5A). To investigate the correlation between electrophoretic mobility and surface localization, whole cell immuno-dot blots were performed with JAR4 except for isolate 4 which JAR4 failed to recognize. For this isolate, polyclonal anti-FHbp sera was used. The median level of surface expression was highest for class 1 isolates and decreased progressively from class 3 isolates (SNP2 only), to class 4 isolates (SNP2 and SNP3) to class 2 isolates (SNP1 and SNP2) (Figure 5B). Overall, nonclass 1 isolates demonstrated a significant 2.2 -fold lower surface expression of FHbp than class 1 isolates (excluding isolate 4) (Figure 5B). These results indicate a failure to cleave the SP decreases the efficiency, but does not abrogate, localization of FHbp to the surface. This data support our findings for recombinant strains of L91543 (Figure 3). In contrast to the general pattern, three isolates (L91543, 12 and 13) demonstrated the presence of FHbp by Western immunoblot but no localization to the cell surface by immuno-dot blot (Figure 5B). The cause of this is explored later in this manuscript.

Interestingly all strains shown to express uncleaved FHbp in this study carry SNP2: substitution of the polar T residue with a hydrophobic A residue at position 19. The importance of polar residues in the h-region of the SP of bacterial lipoproteins first came to light in the late 1970s. In all but one of the 26 bacterial SPs known at that time, polar residues; serine (S) and/or T were found in the h-region and within close proximity to the signal peptidase cleavage site (Vlasuk et al., 1984). A T16A mutant within the SP of E. coli Lpp lipoprotein affected the rate of processing with accumulation of unmodified precursor in the membrane fraction (Vlasuk et al., 1984). It was postulated that this $\mathrm{T}$ residue either directly affected recognition by Lgt or LspA or affected IM translocation hindering accessibility of the SP by these enzymes. We questioned whether the meningococcal Lgt and LspA enzymes fail to recognize FHbp with class 2 (SNP1 and SNP2), class 3 (SNP2), and class 4 (SNP2 and SNP3) SPs (Table 1) and how these preprolipoproteins localize to the surface.

\section{FHbp With SP SNPs Localize to the Surface via Lnt and Slam With Escape From Processing by Lgt and LspA}

To test if strains with SP SNPs in FHbp are affected in lipid modification, one isolate representative of each SP class was tested for the presence of the lipid moiety. These included MC58 (class 1), L91543 (class 2), isolate 6 (class 3) and isolate 18 (class 4) (Tables 1, 2). The strains were grown in medium containing palmitic acid alkyne, FHbp was immuno-precipitated then conjugated (clicked) with biotin azide, which attaches exclusively to proteins carrying this alkyne. Western immunoblot analyses were performed separately with streptavidin and with JAR4. A band of the expected mobility for cleaved FHbp was detected by streptavidin for MC58 clicked with biotin azide and not for the corresponding non-clicked control as expected (Figure 6A). However, no bands were detected for L91543, isolate 6 or 18 whether clicked or non-clicked, suggesting that the FHbp molecules of these three isolates are not lipidated. The absence of lipid by Lgt activity would explain the subsequent failure of LspA to cleave the SP of these isolates (Figure 5A). A recent study in E. coli showed that prevention of diacylglyceryl modification at the conserved cysteine residue of Lpp, by Lgt depletion, prevented Lsp from processing the SP as inferred from its electrophoretic mobility (May et al., 2019). This supports earlier studies that demonstrated that substitution of the Cys residue of Lpp prevented diacylglyceryl modification with accumulation of Lpp exclusively of slower mobility and of the size expected for the unprocessed preprolipoprotein (Inouye et al., 1983). 


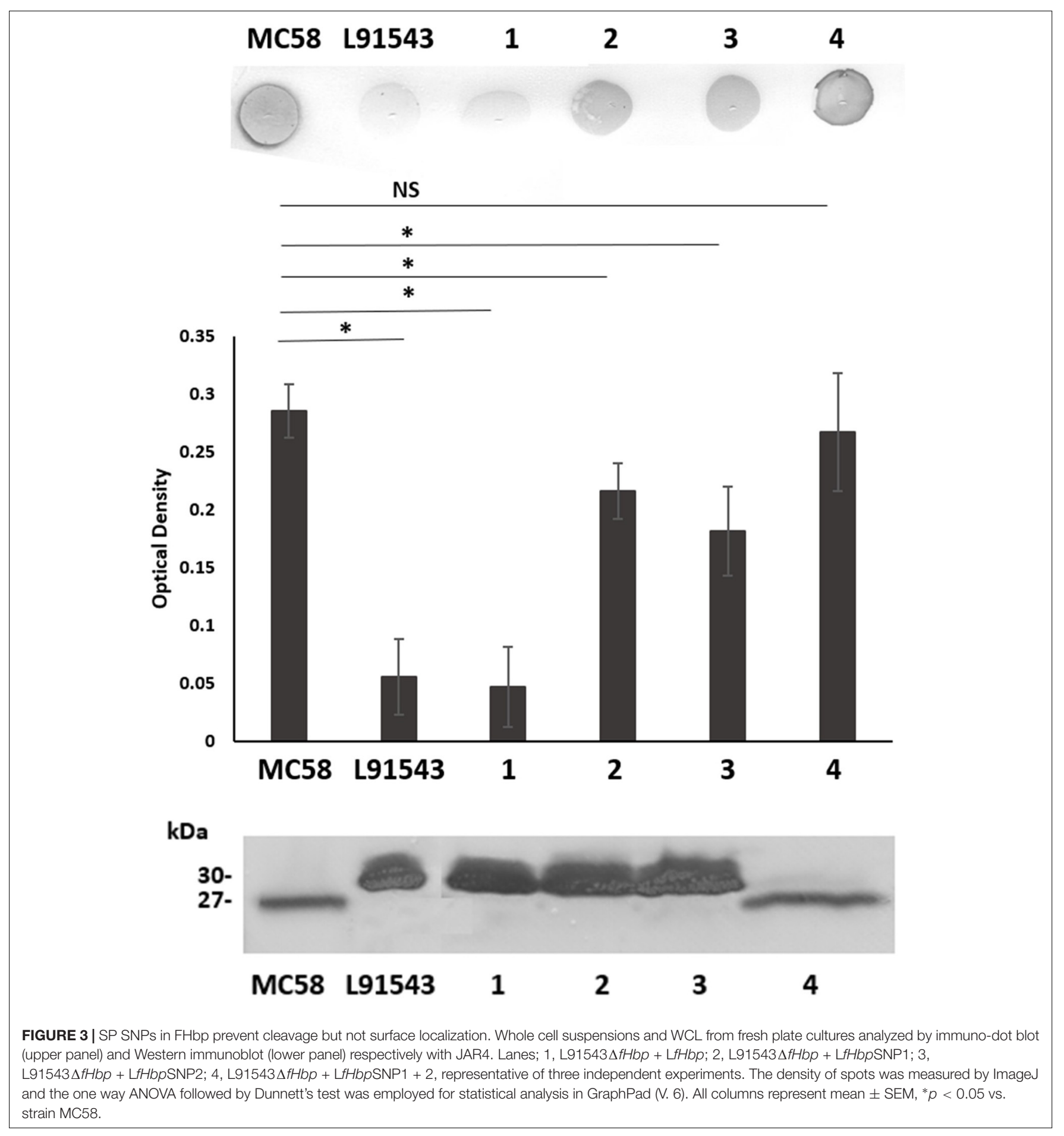

Following failure of Lgt and LspA to lipidate and process FHbp in these non-class 1 strains, we expected the remaining canonical pathway not to be further employed and for the preprolipoprotein to be translocated to the $\mathrm{OM}$ and surface exposed by other mechanisms. To verify discontinuation of the canonical pathway, the lnt gene of the four strains was disrupted. Other than for the L91543 isogenic strains which both showed the expected low level of FHbp, the Lnt mutant version of the other three strains showed a significant reduction in surface localization as well as diminished total FHbp (Figure 6B). This supports our previous study where we showed that MC58Lnt employs mechanisms to reduce FHbp levels thereby preventing the toxic accumulation of the diacylated lipoprotein in the periplasm (da Silva et al., 2017). Our data thus suggests that in addition to the established role of Lnt in triacylating the $\mathrm{N}$-terminal Cys residue in class 1 strains with intact SP (as shown 


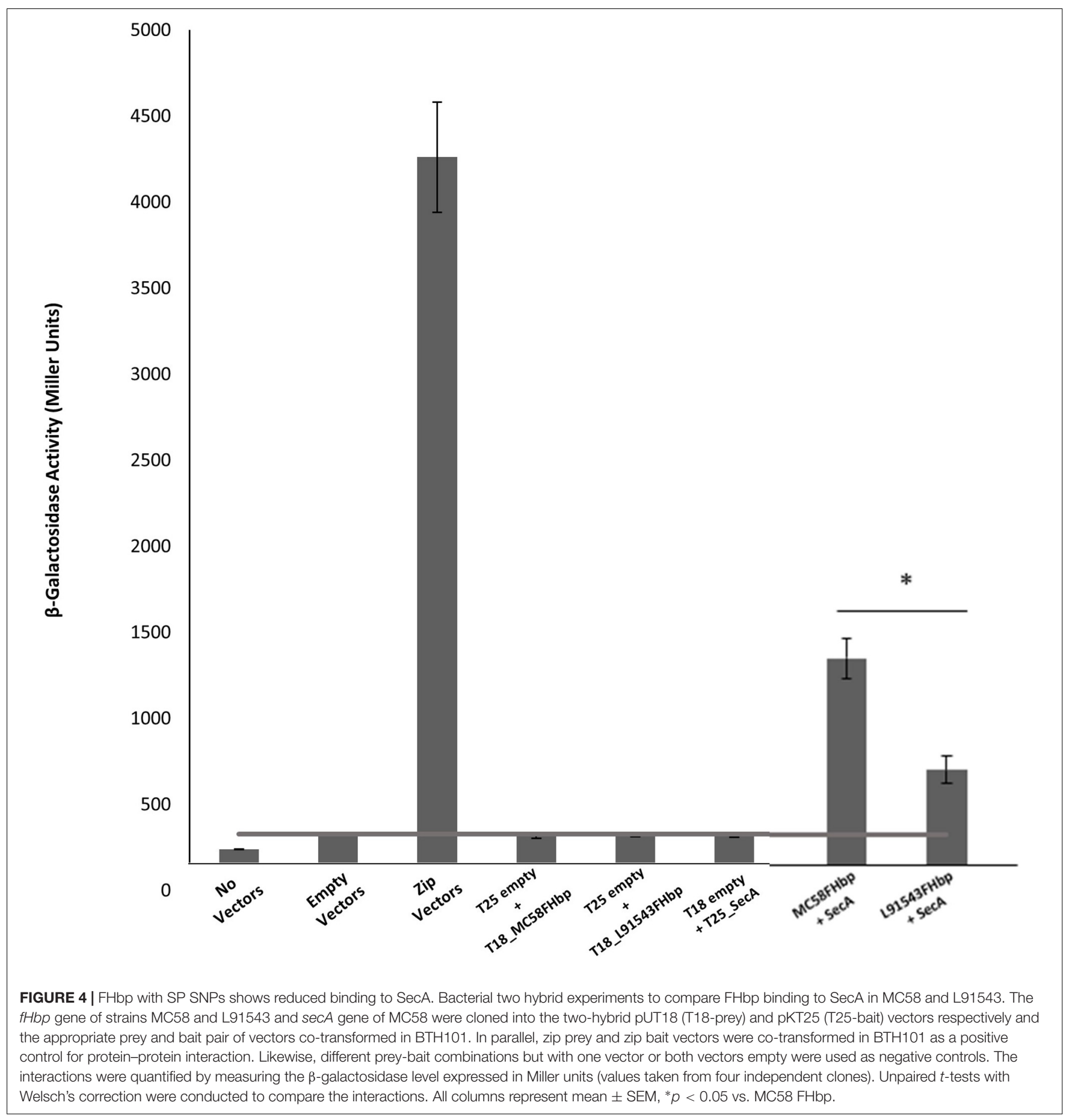

for MC58) (da Silva et al., 2017), Lnt plays a role in delivering FHbp to the OM without the requirement for prior processing by LspA and Lgt.

Given that the canonical pathway appears to resume by Lnt, we questioned whether Lol was employed to translocate FHbp of these strains to the OM however it was not possible to mutate any of the Lol-encoding genes. We next investigated if Slam plays a role in the surface localization of FHbp in these strains. Again with the exception of the poor
FHbp expressor, L91543, the disruption of Slam resulted in significantly reduced surface localization of $\mathrm{FHbp}$ as well as some reduction in total FHbp (Figure 6C). This suggests that Slam plays a role in surface localization of $\mathrm{FHbp}$ regardless of whether FHbp is processed or not. This contradicts what was proposed by Hooda et al. (2016) that Slams are responsible for transport of surface lipoproteins that possess a lipid anchor that needs to be flipped from the inner leaflet to the outer leaflet of the OM (Hooda et al., 2016). Rather, in this study 


\section{A $\mathrm{kDa}$}
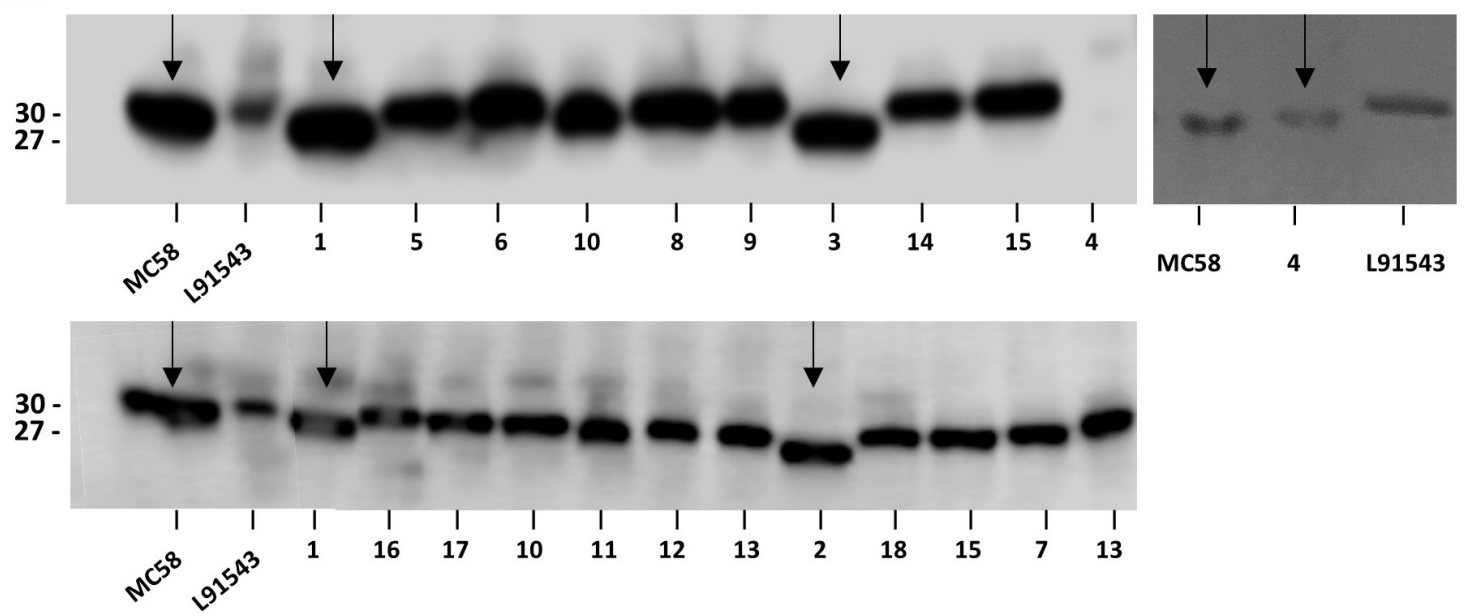

B
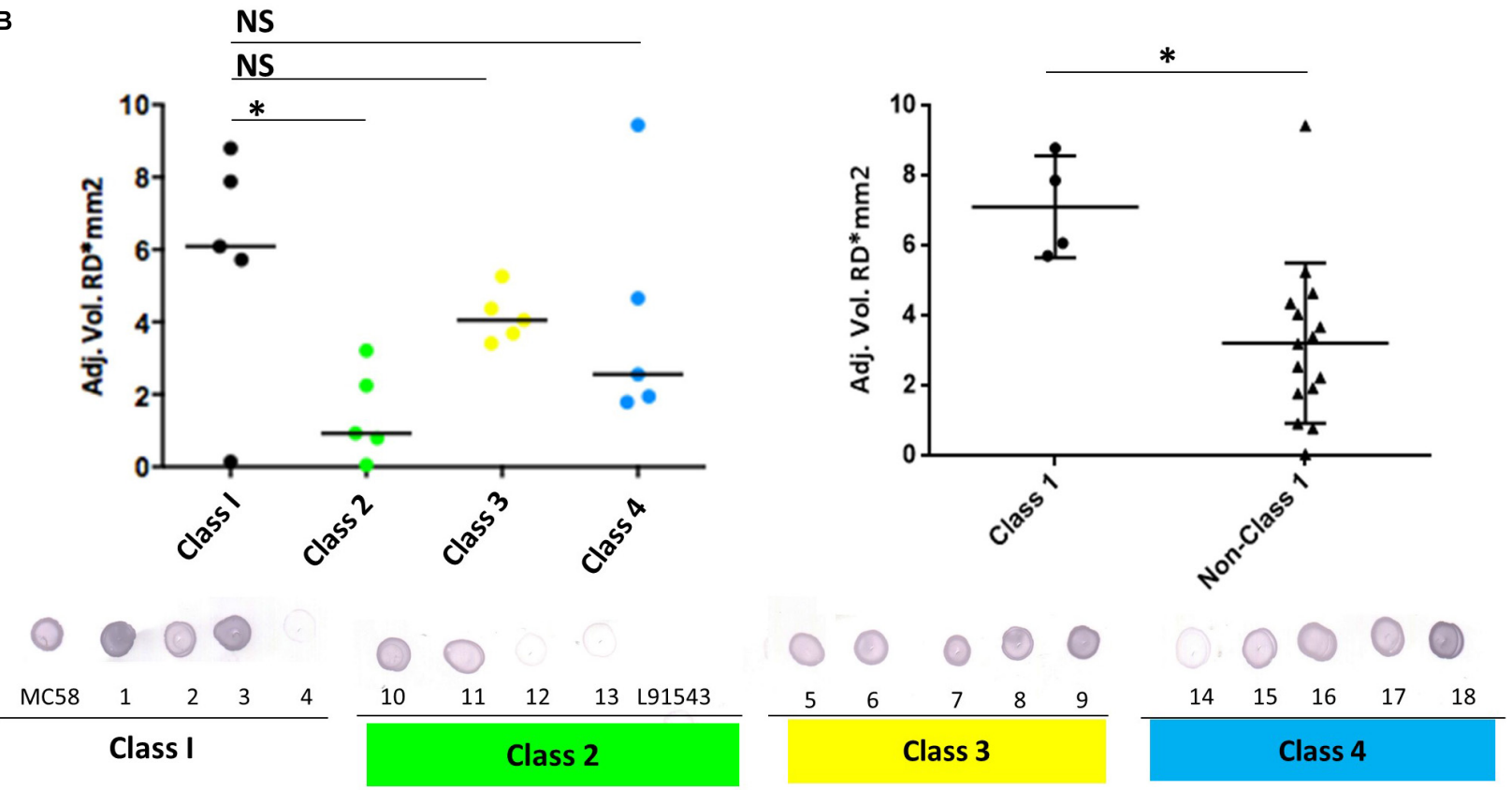

FIGURE 5 | Comparison of FHbp size and surface localization in clinical isolates. For all non-class 1 isolates, the SP is uncleaved yet for most of these isolates, FHbp is surface localized. WCL or whole cell suspensions were prepared from fresh plate cultures and JAR4 used except where indicated. (A) Western immunoblot. For isolate 4, polyclonal anti-FHbp antibody was also used. Arrows indicate faster mobility FHbp. (B) Whole cell immuno-dot blot. The density of spots was measured by ImageJ and the one way ANOVA followed by Dunnett's test employed for statistical analysis in GraphPad (V. 6). All columns represent mean \pm SEM, ${ }^{*} p<0.05$ vs. class 1 . Pooled data of class 1 and non-class 1 were analyzed using un-paired $t$-tests with Welsch's correction. Both columns represent mean \pm SEM, ${ }^{*} p<0.05$ vs. class 1 .

we show that surface localization by Slam is not restricted to lipoproteins.

Given our finding that FHbp with SP SNPs binds less well to SecA, affecting its translocation efficiency to the IM, yet can be translocated to the OM and subsequently surface localized by Slam, we would expect this to be reflected in the sub-cellular distribution of FHbp. In MC58 a processed FHbp protein of $27 \mathrm{kDa}$ was found in the IM, periplasm and $\mathrm{OM}$ but was barely detectable in the cytoplasm, providing strong evidence of highly efficient translocation from cytoplasm to the IM but less efficient translocation to the OM (Figure 6D). In contrast, for isolates 6 and 18, unprocessed precursor was detected in all four cellular compartments; cytoplasm, IM, periplasm and OM, and at high levels in all (Figure 6D). These findings demonstrate that the efficiency of translocation from cytoplasm to IM is reduced in these isolates supporting our BACTH data (Figure 4) but their OM translocation efficiency is comparable with that of MC58. For strain L91543, the total, unprocessed precursor FHbp was noticeably less abundant and all of this localized to the IM and periplasm suggesting complete translocation across 
A

kDa
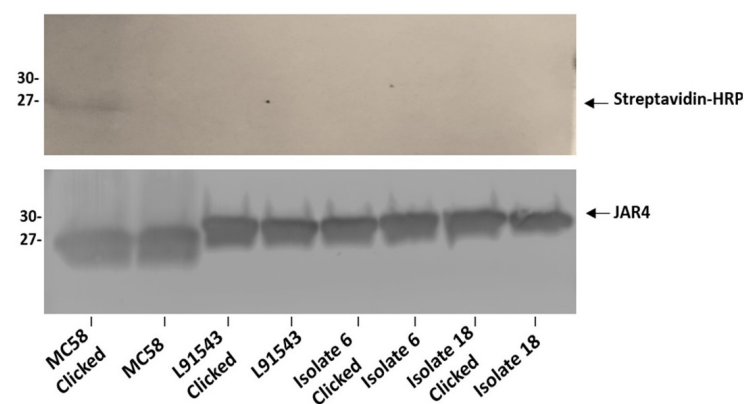

B
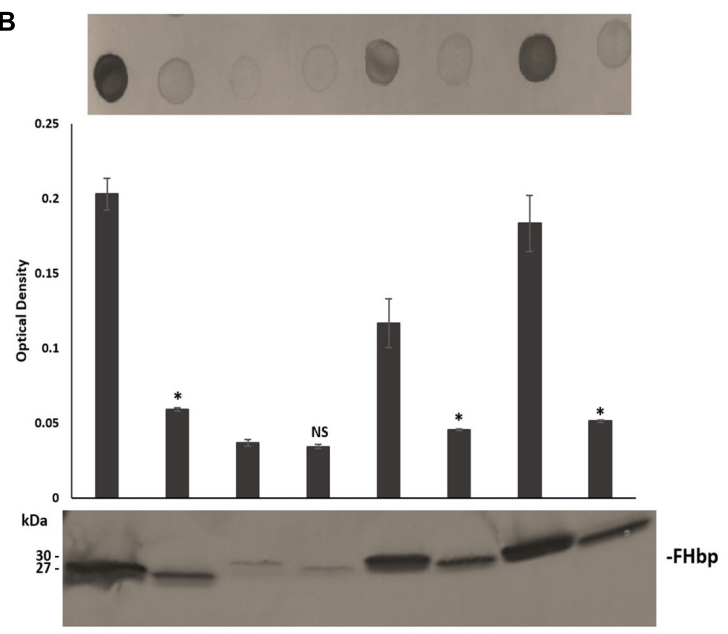

38.

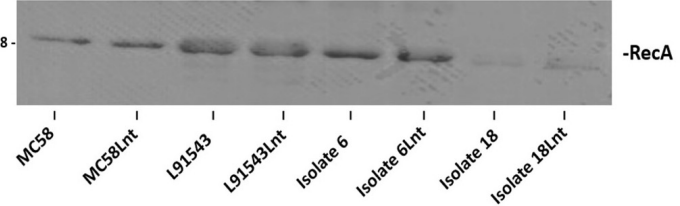

D

C

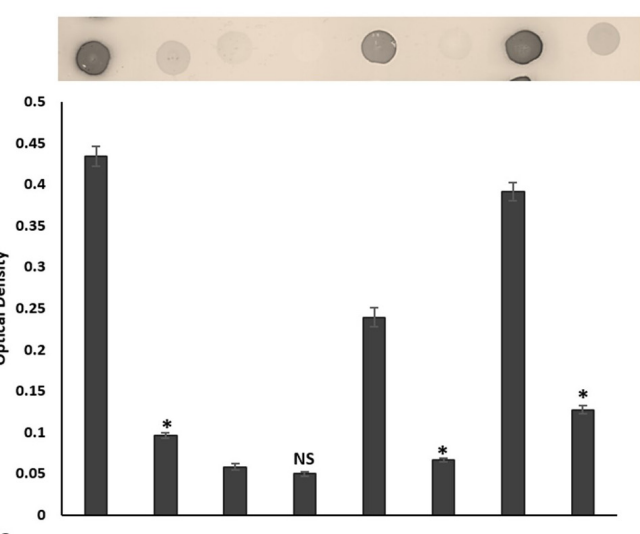

$\mathrm{kDa}$

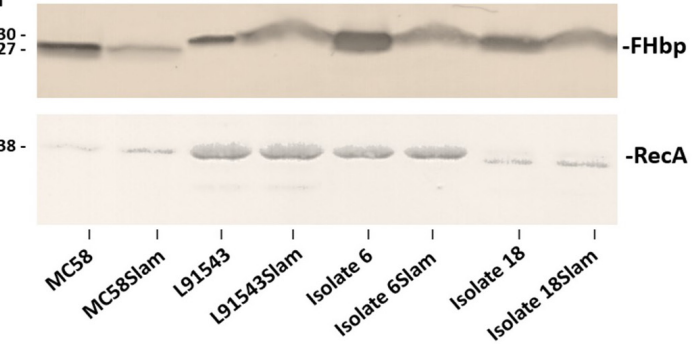

KDa

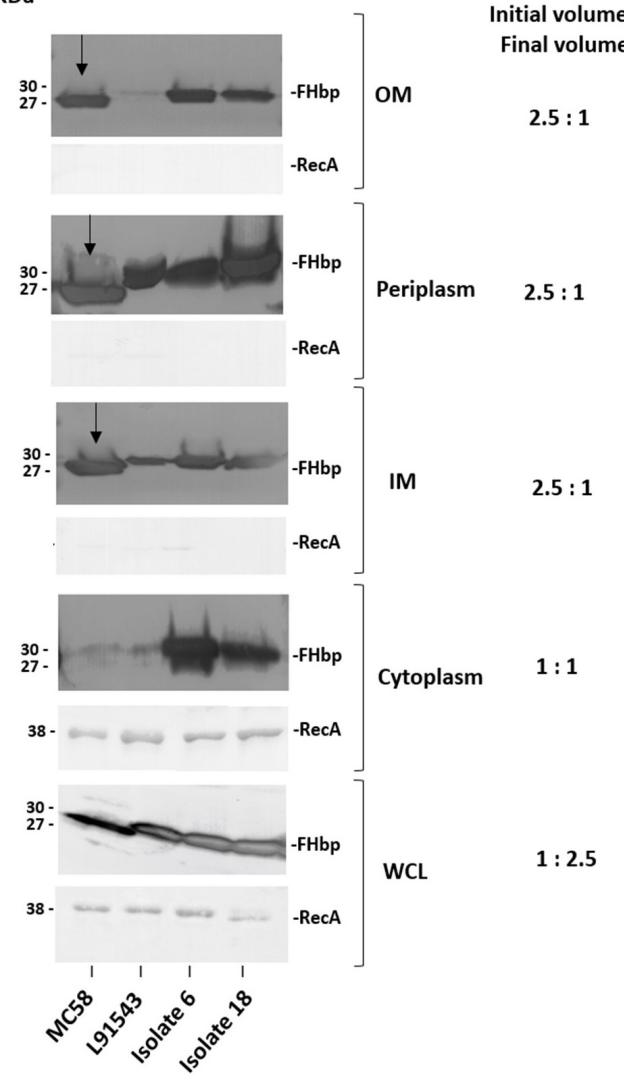

FIGURE 6 | FHbp with SP SNPs localize to the surface via Lnt and Slam with escape from processing by Lgt and LspA. (A) Western immunoblot after growing strains in palmitic acid alkyne and clicking immuno-precipitated FHbp with biotin-azide; Streptavidin-HRP (upper panel) and JAR4 (lower panel). (B,C) Immuno-dot blot of broth cultures $\left(A_{600}\right.$ 0.5) and Western immunoblot of WCL with JAR4. For immuno-dot blot densitometer values, unpaired $t$-tests with Welsch's correction were conducted to compare the isogenic strains. All columns represent mean $\pm \mathrm{SEM},{ }^{*} p<0.05$ vs. wild type isogenic strain. (D) Western immunoblot of different cellular fractions with JAR4. (Lane 1, MC58; 2, L91543; 3, isolate 6; 4, isolate 18.) (B-D) Equal protein loading was confirmed by the determination of RecA protein in each sample. 
the IM (Figure 6D). Conversely, for isolates 6 and 18, the greater overall abundance of the unprocessed precursor appears to have exceeded the capacity of SecA to fully translocate it. From this, we hypothesize that when the unprocessed precursor is abundant, SecA which is already hampered in its binding affinity for non-class $1 \mathrm{FHbp}$, translocates only a portion of this preprolipoprotein to the IM.

\section{Transcript Level of $f H b p$ Influences Sub-Cellular Distribution and Surface Localization of FHbp}

We speculated that the poor expression of the FHbp preprolipoprotein in L91543 was due to weak transcription of $f H b p$, given that strains are known to vary in their transcription efficiency for $f H b p$ (Oriente et al., 2010; Sanders et al., 2012; Cayrou et al., 2018). To test this, RNA was prepared from $A_{600}$ 1.0 broth cultures of all 20 strains and RT-PCR performed with invariant $f H b p$-specific primers. L91543 exhibited 5.8-fold reduction in $\mathrm{fHbp}$ transcript level, and a reduction of 4.6-fold was observed for isolates 12 and 13, compared to MC58 (Figure 7). These three isolates were the same isolates previously found to display FHbp poorly at the cell surface (Figure 5B) hence transcription efficiency influences surface abundance as expected.

To test our hypothesis that an abundance of non-class 1 FHbp exceeds the capacity for complete translocation across the IM, strong expression of fHbp was induced in L91543 and changes in the subcellular distribution of FHbp were investigated. This was made possible by exploiting strain L91543 $\triangle f H b p+\mathrm{L} f H b p$ wherein this class $2 f H b p$ is under the control of the inducible lac $Z$ promoter. First, the amount of surface FHbp preprolipoprotein was compared by immunodot blot between isopropyl- $\beta$-D-thiogalactopyranoside (IPTG)induced and non-induced L91543 $\Delta f H b p+\mathrm{L} f H b p$, relative to the L91543 parent strain and to MC58. The induced recombinant strain localized unprocessed FHbp to the surface with a similar level to that of MC58 (Figure 8A). In the absence of induction, the preprolipoprotein localized to the periplasm and IM but very poorly to the OM (Figure 8B) similar to that observed for the L91543 parent strain (Figure 8B). Upon induction, not only did the quantity of preprolipoprotein dramatically increase, as expected, but as with isolates 6 and 18, some was retained in the cytoplasm while the remainder localized to the IM, periplasm and OM (Figure 8B). Together the data suggest that a certain threshold level of FHbp in the periplasm is tolerated, as shown for strain L91543 (Figure 6D) and non-induced recombinant strain, L91543 $\Delta f H b p+$ LfHbp (Figure 8B). However, overaccumulation in the periplasm appears to force translocation to the OM as observed for induced L91543 $\Delta f H b p+\mathrm{LfHbp}$ and for isolates 6 and 18 (Figures 6D, 8B).

\section{Comparison of Biological Activities of Unprocessed and Processed Surface FHbp}

The binding of FHbp to $\mathrm{fH}$ is vital for the meningococcus to inhibit bacteriolysis by the alternative pathway (Schneider et al., 2006). Crystal structure studies reveal that FHbp folds to form an $\mathrm{N}$-terminal $\beta$ barrel and a C-terminal $\beta$ barrel and that the FHbp-fH complex is held together by extensive interactions between both $\beta$-barrels and domains 6 and 7 of $\mathrm{fH}$ (Schneider et al., 2009). We questioned whether the inclusion of the $26 \mathrm{AA} \mathrm{SP}$ at the $\mathrm{N}$ terminus of FHbp affects the binding of FHbp to $\mathrm{fH}$. Equivalent cell numbers of each of the 20 isolates were incubated with $\mathrm{fH}$. Anti-fH antibody binding was then measured by Fluorescence-Activated Cell Sorting (FACS). No significant difference in binding of class 1 versus non-class 1 isolates was found.

Since a certain threshold level of FHbp on the surface of cells is required for successful killing by vaccine-induced antibodies (Jiang et al., 2010; McNeil et al., 2018) we questioned whether isolates with non-class 1 FHbp SP that typically display less unprocessed FHbp at the surface may be more resistant to killing by antibodies. First FHbp surface expression was rigorously quantified by FACS analysis with JAR4. Since JAR4 is a monoclonal antibody binding a single epitope (Supplementary Table S1), saturation of meningococcal cells with this antibody should result in the maximal binding of one antibody per FHbp molecule. Commercially available microspheres were used to quantify the number of antibodies bound per cell $(A B C)$ and enable us to infer from this the number of FHbp molecules displayed per cell. The SBA assay was performed using the FHbp-specific monoclonal antibody JAR5 along with JAR4. Cooperativity between these two antibodies is necessary to elicit bactericidal activity in the presence of human complement (Beernink et al., 2008; Welsch et al., 2008; Malito et al., 2016). JAR5 is known to bind the FHbp epitope comprising AA residues 84-91 and 115-123 (Malito et al., 2016) (Supplementary Table S1). Since MC58 and L91543 were previously shown to contrast starkly in their FHbp exposure and susceptibility to killing by FHbp-immunized sera in SBA assays (Newcombe et al., 2014) these strains were used as positive and negative controls respectively to benchmark all other isolates.

We first noted that isolates 4, 12, and 13 displayed very poor surface FHbp at a level comparable with that of L91543 (Figure 9A) supporting our previous immuno-dot blot data for these isolates (Figure 5B). These levels were well below 757 molecules per cell, the threshold level for killing by anti-FHbp antibodies (Biagini et al., 2016). Unsurprisingly, extremely weak bactericidal activity was detected for these isolates in SBA assays with up to $11 \%$ killing demonstrated (Figure 9B). Our findings are explained by the poor transcript levels observed for isolates L91543, 12, and 13 (Figure 7) and by failure of JAR4 to bind isolate 4 (Figure 5B). Pooled class 1 isolates were compared with pooled non-class 1 isolates after discounting the four outliers. For ABC, the average was 7869 vs. 4547 molecules $(p=0.0038)$ and for SBA the average was 73.7 vs. $53.4 \%(p=0.0009)$ (Figure 9C).

\section{DISCUSSION}

Since the discovery of FHbp as a lipoprotein (Fletcher et al., 2004) it has been assumed that all meningococci that express FHbp synthesize the mature lipoprotein. The failure to process FHbp including lipidation and SP cleavage, in strain L91543 was 


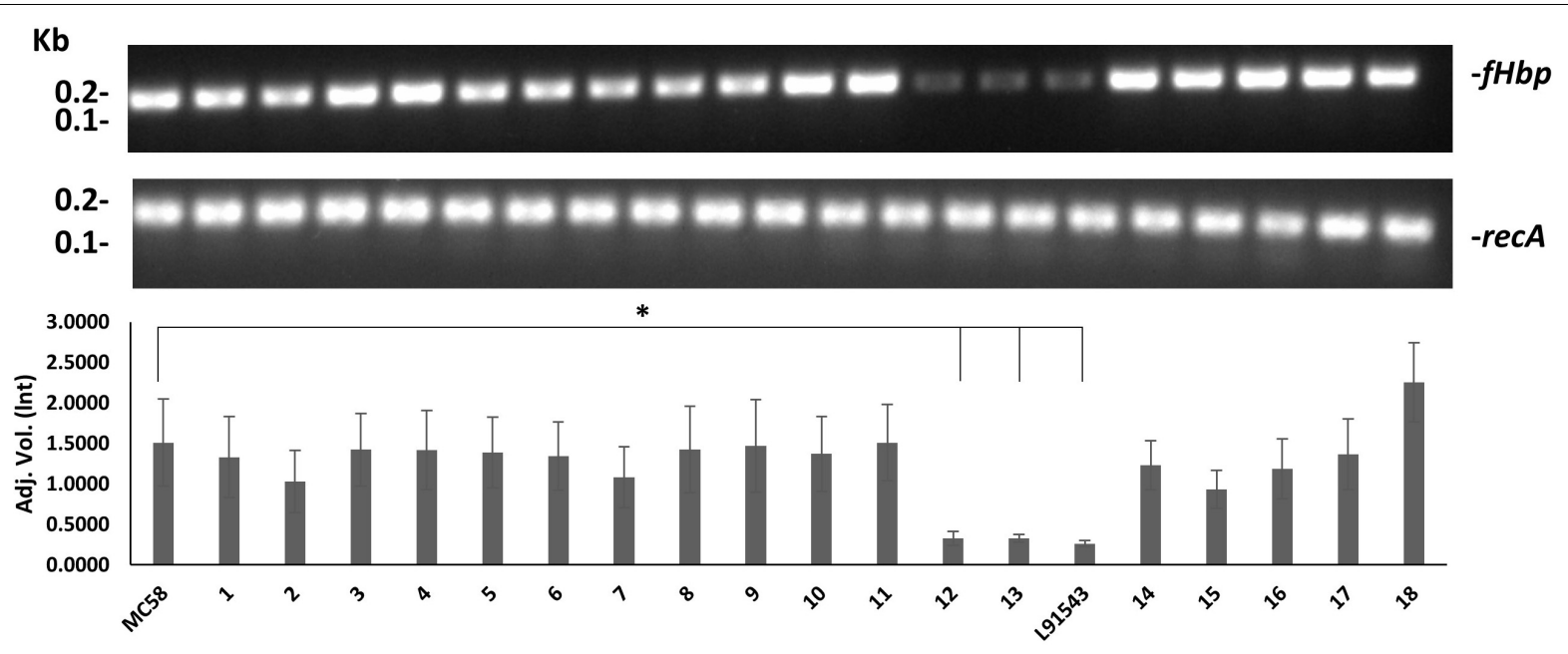

FIGURE 7 | Comparison of $f H b p$ transcript level between strains. RT-PCR of $f H b p$ and recA. One representative experiment of 3 is shown. Band intensity was measured using Image lab v4.0.1 and the data acquired using Linear non-threshold model (Int). The one-way ANOVA followed by Dunnett's test was employed for statistical analysis in GraphPad (V. 6). All columns represent mean \pm SEM, * $p<0.05$ vs. strain MC58.

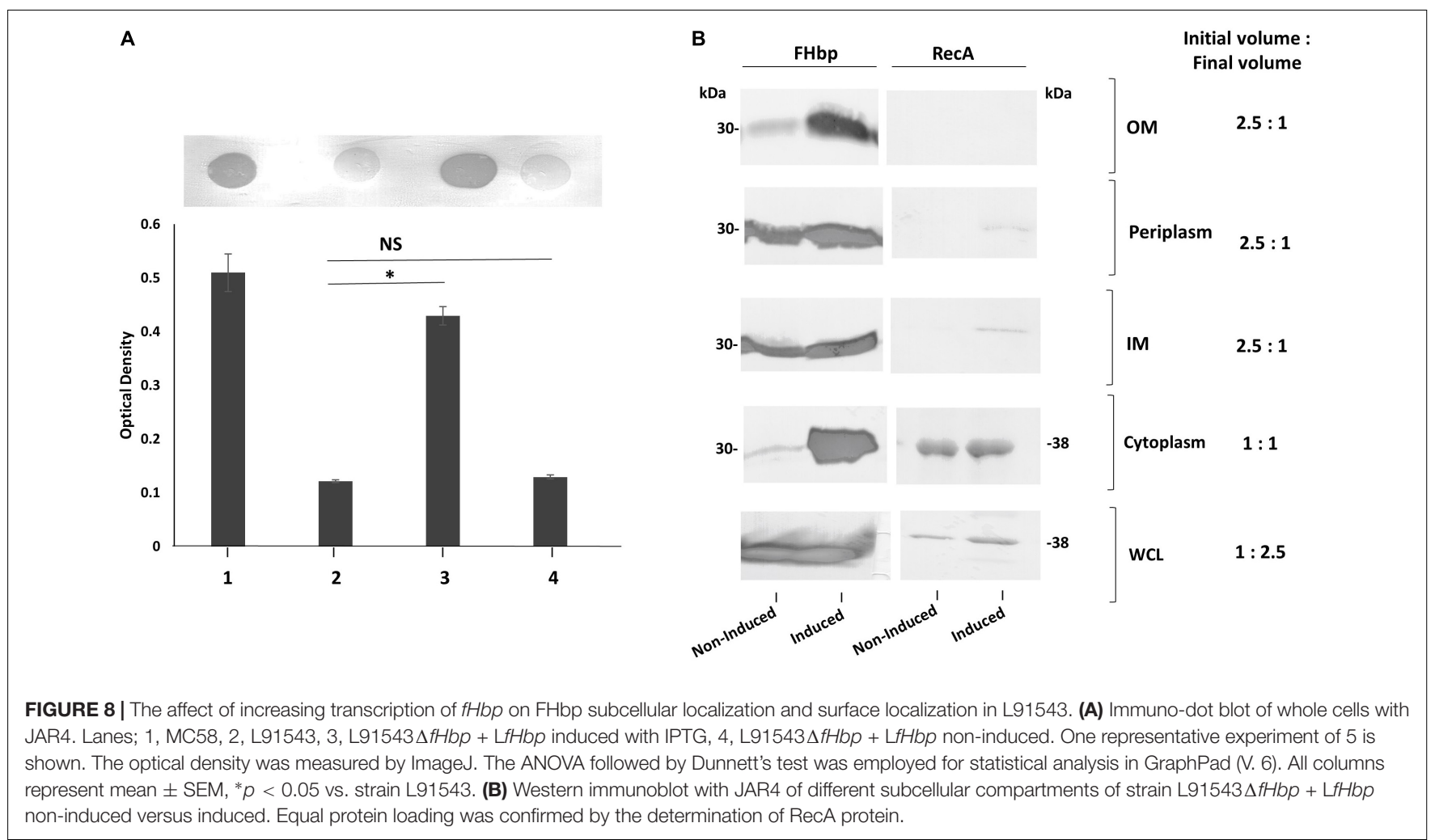

directly linked to mutations in the SP (L15F) and (T19A) that interrupt Sec-mediated translocation. The correlation between SP mutations and failure to process FHbp was further confirmed in a panel of invasive group $B$ isolates. Remarkably the preprolipoprotein of isolates possessing variant SPs localized to the cell surface but with twofold lower abundance and in the subset of isolates tested, this was attributed to its partial retention in the cytoplasm. For L91543 with poor transcription of $f H b p$, the less abundant preprolipoprotein was all translocated across the IM but none was translocated to the OM for subsequent surface display. This could however be overcome by increasing the transcription strength of $f H b p$.

It is known that Sec-dependent proteins are exported in unfolded states to the Sec-YEG channel (Figure 1) as this channel is only large enough to accommodate unfolded proteins ( $\mathrm{Li}$ et al., 2016). SecA acts promptly to bind nascent polypeptides as they emerge from the ribosome during translation and maintains the polypeptide in an unfolded state (Huber et al., 

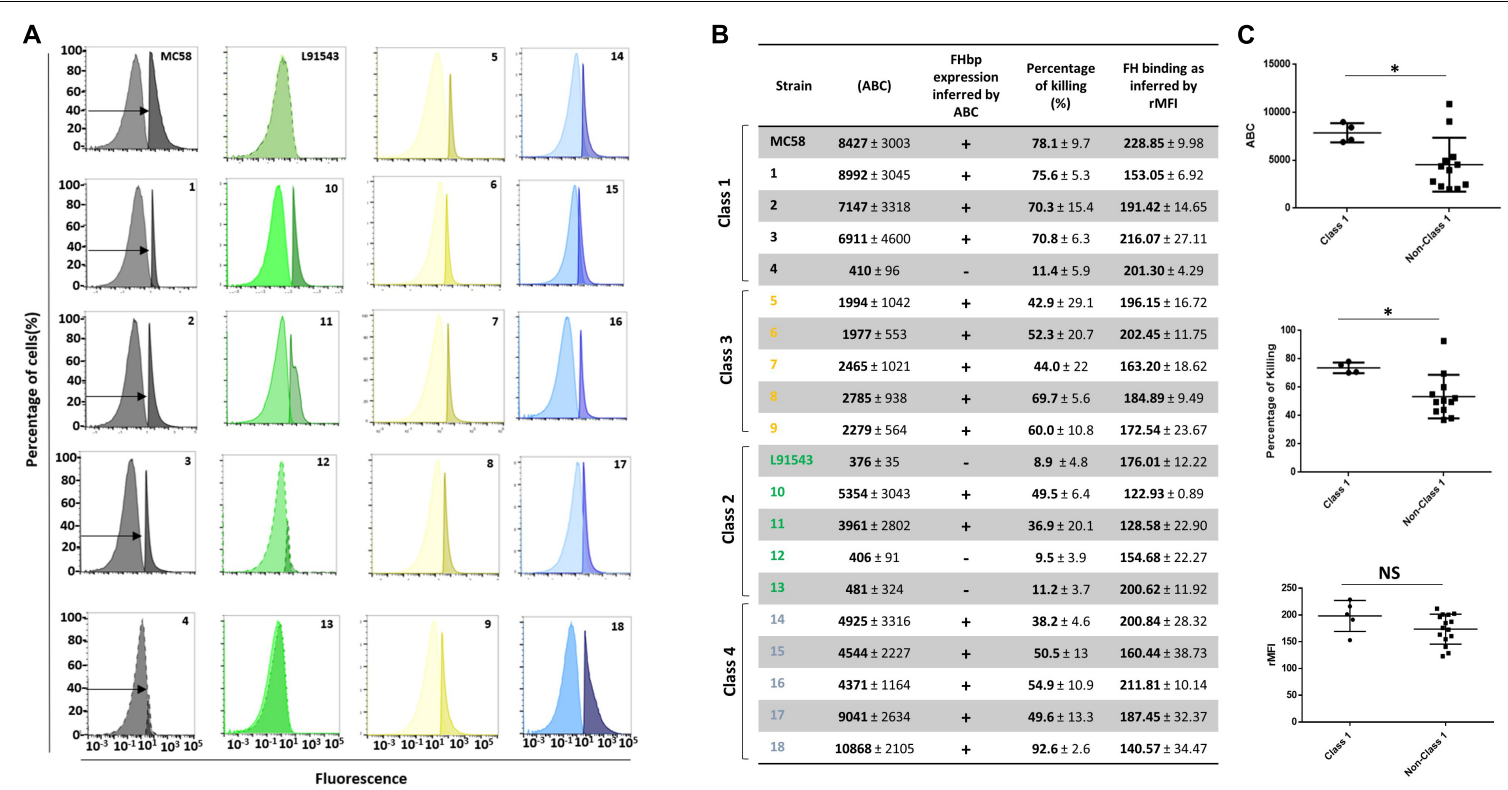

FIGURE 9 | Comparison of biological activities of unprocessed and processed surface-localized FHbp. (A) FACS analysis with Mab JAR4. A representative flow cytometry plot for each isolate is shown. The read-outs from negative control samples, cells incubated with secondary antibody only (left peak) were gated (as shown by the arrows). The read-outs from samples incubated with both primary and secondary antibody were overlaid. (B) The number of JAR4 antibody molecules bound per cell $(\mathrm{ABC})$ and corresponding prediction for successful killing in SBA assays is denoted by +. The actual percentage killing for each isolate with Mabs JAR4, JAR5 and human complement is shown. The mean ABC for each isolate was derived from three independent FACS experiments and the mean percentage killing derived from four independent SBA experiments, each with two technical replicates. The binding of fH to cells. Values show the Relative Mean of Fluorescence Intensity (rMFI) for total fluorescing cells after incubation with $\mathrm{fH}$ and anti-fH PE-conjugated antibody. The values were normalized against the negative control of cells alone incubated with antibody. (C) Pooled data for class 1 and non-class 1 isolates, excluding the outliers. Values were analyzed by unpaired $t$-tests with Welsch's correction. Columns represent mean $\pm \mathrm{SEM},{ }^{*} p<0.05$ vs. class 1.

2017). Disrupting this interaction causes a partial defect in SecA-mediated translocation (Huber et al., 2017) and reduced efficiency of transport to the IM due to the propensity for the preprolipoprotein to fold and become incompetent for translocation (Steiner et al., 2006). Based on new insights in SecA-mediated translocation (Mao et al., 2016), it is possible that the preprolipoprotein that is translocated is not orientated correctly in the IM such that Lgt fails to recognize this FHbp precursor as substrate.

Our findings suggest that the polar AA, threonine (T), four AAs away from the lipobox of FHbp, is the most critical for correct translocation by SecA in MC58 as the T19A mutation is carried by all non-class 1 isolates (at least $88 \%$ of MRF isolates) and is the sole mutation in class 3 isolates ( $48 \%$ of MRF isolates) (Table 1). This supports the work of Vlasuk and co-workers who in the early 80 s demonstrated the importance of polar residues close to the lipobox of E. coli Lpp, for translocation (Vlasuk et al., 1984). We postulate that in T19A FHbp variants, the reduced efficiency of binding to the SP by SecA results in the propensity for folding with consequent retention of some of this translocation-incompetent preprolipoprotein in the cytoplasm. This retention was evident for both isolates 6 and 18 tested that express an abundance of the preprolipoprotein and for L91543 after induction of strong expression of FHbp (Figures 6D, 8B). The remaining preprolipoprotein was translocated across the IM and some of this was translocated to the $\mathrm{OM}$ and further surface-displayed. It is plausible that the SP anchors FHbp to the
OM facilitated by its hydrophobic region. Upon accumulation of the unprocessed precursor FHbp in the IM beyond a threshold level, translocation to the $\mathrm{OM}$ is forced, probably to prevent clogging which would prohibit the translocation of other OMdestined proteins and be potentially fatal for the cell. We provide data suggesting this translocation is partly mediated by Lnt highlighting a secondary role for Lnt as a chaperone facilitating delivery to Lol. We further show that localization to the surface involves Slam revealing that Slam is not lipoprotein-specific. The broader specificity of Slam, than originally thought, for OM protein localization is plausible given Slam recognizes the C terminal structure of its substrates (Hooda et al., 2016) which may be shared between lipoproteins and OM proteins.

From analysis of the SP sequence of 1895 invasive group B isolates from the United Kingdom MRF collection, as few as 9\% are predicted to express FHbp as a lipoprotein. Conversely, at least $88 \%$ are predicted to display the unprocessed, precursor at the surface, provided sufficient transcription strength of $f H b p$. A similar prevalence is expected for other isolates circulating around the globe. Given that isolates displaying non-class 1 FHbp SP are far more prevalent than class 1 isolates, there must be a fitness benefit for the former. One explanation for evolutionary selection for the former could be that exportation of unprocessed FHbp is less metabolically costly than employing processing enzymes to modify FHbp prior to localization of the mature lipoprotein to the surface. Alternatively the significantly reduced surface exposure of the preprolipoprotein, shown in 
this study, may be selected for to reduce anti-FHbp antibodymediated killing whilst retaining sufficient $\mathrm{fH}$ binding. It is worth noting here that we would anticipate seeing even greater differences in SBA responses between Class 1 and non-Class 1 isolates using sera from Trumenba-vaccinated individuals (as opposed to the two Mabs used in this study) since this vaccine specifically targets SP-cleaved, lipidated FHbp (Class 1 FHbp) with polyclonal antibodies generated to multiple epitopes.

To summarize, our results reveal that the AA sequence requirement for lipoprotein SPs to direct translocation to the IM completely and correctly for processing to follow, are more constrained than previously thought. In the DOLOP database of predicted bacterial lipoproteins https://www.mrclmb.cam.ac.uk/genomes/dolop/predicted/ab.shtml (Babu et al., 2006) the algorithm does not include the requirement for the polar residue and for many of the proteins in this database, this residue is absent. These proteins are unlikely to undergo maturation to become lipoproteins and thus the size of bacterial lipoproteomes could be over-estimated. More experimental work is required for other bacterial lipoproteins to fully determine the structural features, in particular, critical AAs, that are required for lipoprotein maturation and localization. From this, new programs with greater prediction power can be deployed which would be of importance to scientists developing vaccines based on bacterial lipoproteins, a growing area of research.

Finally, our finding that the FHbp vaccine target is predominantly unprocessed FHbp as opposed to cleaved, lipidated FHbp, is particularly important for Trumenba which is composed exclusively of 2 lipidated variants of FHbp. Altering the vaccine to contain uncleaved, non-lipidated FHbp should improve its target specificity. It is unknown to the public which reference strains are used in Pfizer's MEASURE assay (Meningococcal Antigen Surface Expression) (Donald et al., 2017) as standards for quantifying FHbp surface abundance on test isolates in order to predict vaccine coverage. With knowledge of the prevalence of $4 \mathrm{SP}$ classes for FHbp - the majority of isolates expressing non-class 1 , the choice of reference strains should be re-considered to ensure sufficient representation across these classes. In predicting coverage by Trumenba, knowledge on the nucleotide level of promoter and upstream regulatory sequences (Cayrou et al., 2018) and on the AA level of the SP sequence are both key as the former dictates FHbp expression level and the latter its sub-cellular distribution and importantly surface localization. This sequence information will enable an improved understanding of Trumenba vaccine escape strains and this knowledge can feed into developing improved formulations. Our findings also bear relevance for Bexsero although this vaccine is multivalent and thus less heavily reliant on the successful targeting of FHbp. Our study highlights the importance of multivalent vaccine approaches.

\section{DATA AVAILABILITY STATEMENT}

The FACS data discussed in this publication have been deposited in FlowRepository (Spidlen et al., 2012) and are available through accession numbers: FR-FCM-ZYTU,
http://flowrepository.org/id/RvFrLUcAM69ZrTziXZJn622NsvQ gwRuVqoRC28XA9wBxR2KZsAJ2yym5ToAfMt6p, FR-FCM-Z YTV, http://flowrepository.org/id/RvFryhGbJHv5UMqqZ1bEfLE z6aO8QEV0EsQtjKdcSjlMt1rCtFIiT9HmOrMZYBms and FRFCM-ZYUZ, http://flowrepository.org/id/RvFraaVCkdcJJYpYJO c4CFQNbETeVGwTy1bcNwXpA3YotemW9trGUDNKJFgxXn Ki. The closed genome sequence of L91543 is available under accession number CP016684.

\section{AUTHOR CONTRIBUTIONS}

RS performed all of the experiments, analyzed the data, and contributed to the experimental design and writing of the manuscript (Materials and Methods and Figures). RG devised the project, supervised the experiments, analyzed the data, and wrote the manuscript. AK closed the genome of L91543. NO and KW assisted with the bioinformatics analyses. CB provided the strains and FHbp protein sequences. AR provided technical advice.

\section{FUNDING}

The funding for this work was received from Conselho Nacional de Desenvolvimento Científico e Tecnológico (CNPq) (201521/2015-6) for a 4-year Ph.D. program for RS. Additional funding for consumables was provided by the School of Life Sciences, University of Nottingham as part of a start-up package for RG.

\section{ACKNOWLEDGMENTS}

This publication made use of the MRF Meningococcus Genome Library (http://www.meningitis.org/research/genome) developed by Public Health England, the Wellcome Trust Sanger Institute and the University of Oxford as a collaboration. We thank Professor Nigel P. Minton for bench space and use of equipment, Professor Liz Sockett for proofreading this manuscript, Izadora L. A. Rabelo for assisting with FACS analysis and Marcelo Depólo for helpful discussions about SPs. This manuscript has been released as a Pre-Print at BioRxiv http://biorxiv.org/cgi/content/ short/693374v1.

\section{SUPPLEMENTARY MATERIAL}

The Supplementary Material for this article can be found online at: https://www.frontiersin.org/articles/10.3389/fmicb.2019. 02847/full\#supplementary-material

FIGURE S1 | Western immunoblotting of WCL of L91543 and L91543 $\triangle f H b p$ with JAR4.

TABLE S1 | Multiple alignment of FHbp of isolates used in this study and AA identities across the whole protein relative to MC58. Framed in red and gray boxes are AA residues in the epitopes recognized by JAR4 and JAR5 respectively.

TABLE S2 | AA identity of proteins involved in translocation, processing and surface localization of FHbp in L91543 compared to MC58. 


\section{REFERENCES}

Ames, G. F., Prody, C., and Kustu, S. (1984). Simple, rapid, and quantitative release of periplasmic proteins by chloroform. J. Bacteriol. 160, 1181-1183.

Babu, M. M., Priya, M. L., Selvan, A. T., Madera, M., Gough, J., Aravind, L., et al. (2006). A database of bacterial lipoproteins (DOLOP) with functional assignments to predicted lipoproteins. J. Bacteriol. 188, 2761-2773. doi: 10. 1128/jb.188.8.2761-2773.2006

Basta, N. E., and Christensen, H. (2016). 4CMenB vaccine effectiveness: reasons for optimism. Lancet 388, 2719-2721. doi: 10.1016/s0140-6736(16)32061-x

Beernink, P. T., and Granoff, D. M. (2009). The modular architecture of meningococcal factor H-binding protein. Microbiology 155, 2873-2883. doi: 10.1099/mic.0.029876-0

Beernink, P. T., Shaughnessy, J., Braga, E. M., Liu, Q., Rice, P. A., Ram, S., et al. (2011). A meningococcal factor $\mathrm{H}$ binding protein mutant that eliminates factor $\mathrm{H}$ binding enhances protective antibody responses to vaccination. J. Immunol. 186, 3606-3614. doi: 10.4049/jimmunol.1003470

Beernink, P. T., Welsch, J. A., Bar-Lev, M., Koeberling, O., Comanducci, M., and Granoff, D. M. (2008). Fine antigenic specificity and cooperative bactericidal activity of monoclonal antibodies directed at the meningococcal vaccine candidate factor h-binding protein. Infect. Immun. 76, 4232-4240. doi: 10.1128/ IAI.00367-08

Biagini, M., Spinsanti, M., De Angelis, G., Tomei, S., Ferlenghi, I., Scarselli, M., et al. (2016). Expression of factor $\mathrm{H}$ binding protein in meningococcal strains can vary at least 15 -fold and is genetically determined. Proc. Natl. Acad. Sci. U.S.A. 113, 2714-2719. doi: 10.1073/pnas.1521142113

Brehony, C., Wilson, D. J., and Maiden, M. C. (2009). Variation of the factor H-binding protein of Neisseria meningitidis. Microbiology 155, 4155-4169. doi: 10.1099/mic.0.027995-0

Cayrou, C., Akinduko, A. A., Mirkes, E. M., Lucidarme, J., Clark, S. A., Green, L. R., et al. (2018). Clustered intergenic region sequences as predictors of factor $\mathrm{H}$ binding protein expression patterns and for assessing Neisseria meningitidis strain coverage by meningococcal vaccines. PLoS One 13:e0197186. doi: 10. 1371/journal.pone.0197186

Chambonnier, G., Roux, L., Redelberger, D., Fadel, F., Filloux, A., Sivaneson, M., et al. (2016). The hybrid histidine kinase LadS Forms a multicomponent signal transduction system with the GacS/GacA two-component system in Pseudomonas aeruginosa. PLoS Genet. 12:e1006032. doi: 10.1371/journal.pgen. 1006032

Clark, V. L., Campbell, L. A., Palermo, D. A., Evans, T. M., and Klimpel, K. W. (1987). Induction and repression of outer membrane proteins by anaerobic growth of Neisseria gonorrhoeae. Infect. Immun. 55, 13591364.

da Silva, R. A. G., Churchward, C. P., Karlyshev, A. V., Eleftheriadou, O., Snabaitis, A. K., Longman, M. R., et al. (2017). The role of apolipoprotein $\mathrm{N}$-acyl transferase, Lnt, in the lipidation of factor $\mathrm{H}$ binding protein of Neisseria meningitidis strain MC58 and its potential as a drug target. Br. J. Pharmacol. 174, 2247-2260. doi: 10.1111/bph.13660

da Silva, R. A. G., Karlyshev, A. V., Oldfield, N. J., Wooldridge, K. G., Bayliss, C. D., Ryan, A., et al. (2019). Vaccine antigen, Factor H binding protein, is typically a non-lipidated precursor that localises to the meningococcal surface by Slam. bioRxiv[Pre-Print] . doi: 10.1101/693374

Donald, R. G., Hawkins, J. C., Hao, L., Liberator, P., Jones, T. R., Harris, S. L., et al. (2017). Meningococcal serogroup B vaccines: estimating breadth of coverage. Hum. Vacc. Immunother. 13, 255-265. doi: 10.1080/21645515.2017.126 4750

Driessen, A. J., and Nouwen, N. (2008). Protein translocation across the bacterial cytoplasmic membrane. Annu. Rev. Biochem. 77, 643-667. doi: 10.1146/ annurev.biochem.77.061606.160747

Fletcher, L. D., Bernfield, L., Barniak, V., Farley, J. E., Howell, A., Knauf, M., et al. (2004). Vaccine potential of the Neisseria meningitidis 2086 lipoprotein. Infect. Immun. 72, 2088-2100. doi: 10.1128/iai.72.4.2088-2100.2004

Froger, A., and Hall, J. E. (2007). Transformation of plasmid DNA into E. coli using the heat shock method. J. Vis. Exp. 6, 253. doi: 10.3791/253

Gandhi, A., Balmer, P., and York, L. J. (2016). Characteristics of a new meningococcal serogroup B vaccine, bivalent rLP2086 (MenB-FHbp; Trumenba(R)). Postgrad. Med. 128, 548-556. doi: 10.1080/00325481.2016. 1203238
Gelis, I., Bonvin, A. M., Keramisanou, D., Koukaki, M., Gouridis, G., Karamanou, S., et al. (2007). Structural basis for signal-sequence recognition by the translocase motor SecA as determined by NMR. Cell 131, 756-769. doi: 10. 1016/j.cell.2007.09.039

Giuntini, S., Reason, D. C., and Granoff, D. M. (2011). Complement-mediated bactericidal activity of anti-factor $\mathrm{H}$ binding protein monoclonal antibodies against the meningococcus relies upon blocking factor $\mathrm{H}$ binding. Infect. Immun. 79, 3751-3759. doi: 10.1128/IAI.05182-11

Gouridis, G., Karamanou, S., Gelis, I., Kalodimos, C. G., and Economou, A. (2009). Signal peptides are allosteric activators of the protein translocase. Nature 462, 363-367. doi: 10.1038/nature08559

Hooda, Y., Lai, C. C.-L., Judd, A., Buckwalter, C. M., Shin, H. E., Gray-Owen, S. D., et al. (2016). Slam is an outer membrane protein that is required for the surface display of lipidated virulence factors in Neisseria. Nat. Microbiol. 1:16009. doi: 10.1038/nmicrobiol.2016.9

Horton, R. M. (1995). PCR-mediated recombination and mutagenesis. SOEing together tailor-made genes. Mol. Biotechnol. 3, 93-99. doi: 10.1007/bf02789105

Huber, D., Jamshad, M., Hanmer, R., Schibich, D., Doring, K., Marcomini, I., et al. (2017). SecA cotranslationally interacts with nascent substrate proteins in vivo. J. Bacteriol. 199: e00622-16. doi: 10.1128/JB.00622-16

Inouye, S., Franceschini, T., Sato, M., Itakura, K., and Inouye, M. (1983). Prolipoprotein signal peptidase of Escherichia coli requires a cysteine residue at the cleavage site. EMBO J. 2, 87-91. doi: 10.1002/j.1460-2075.1983.tb01386.x

Jiang, H. Q., Hoiseth, S. K., Harris, S. L., Mcneil, L. K., Zhu, D., Tan, C., et al. (2010). Broad vaccine coverage predicted for a bivalent recombinant factor $\mathrm{H}$ binding protein based vaccine to prevent serogroup B meningococcal disease. Vaccine 28, 6086-6093. doi: 10.1016/j.vaccine.2010.06.083

Karlyshev, A. V., Snyder, L. A., Mcfadden, J., and Griffin, R. (2015). Insight into proteomic investigations of Neisseria meningitidis serogroup C strain L91543 from analysis of its genome sequence. FEMS Microbiol. Lett. 362:fnv055. doi: 10.1093/femsle/fnv055

Kellogg, D. S. Jr., Peacock, W. L. Jr., Deacon, W. E., Brown, L., and Pirkle, D. I. (1963). Neisseria gonorrhoeae I. virulence generically linked to clonal variation. J. Bacteriol. 85, 1274-1279.

Kovacs-Simon, A., Titball, R. W., and Michell, S. L. (2011). Lipoproteins of bacterial pathogens. Infect. Immun. 79, 548-561. doi: 10.1128/IAI.00682-10

Li, L., Park, E., Ling, J., Ingram, J., Ploegh, H., and Rapoport, T. A. (2016). Crystal structure of a substrate-engaged SecY protein-translocation channel. Nature 531, 395-399. doi: 10.1038/nature17163

Loh, E., Lavender, H., Tan, F., Tracy, A., and Tang, C. M. (2016). Thermoregulation of Meningococcal fHbp, an important virulence factor and vaccine antigen, is mediated by anti-ribosomal binding site sequences in the open reading frame. PLoS Pathog. 12:e1005794. doi: 10.1371/journal.ppat.1005794

Malito, E., Lo Surdo, P., Veggi, D., Santini, L., Stefek, H., Brunelli, B., et al. (2016). Neisseria meningitidis factor H-binding protein bound to monoclonal antibody JAR5: implications for antibody synergy. Biochem. J. 473, 4699-4713. doi: $10.1042 /$ bcj20160806

Mao, G., Zhao, Y., Kang, X., Li, Z., Zhang, Y., Wang, X., et al. (2016). Crystal structure of E. coli lipoprotein diacylglyceryl transferase. Nat. Commun. 7:10198. doi: 10.1038/ncomms10198

Mascioni, A., Moy, F. J., Mcneil, L. K., Murphy, E., Bentley, B. E., Camarda, R., et al. (2010). NMR dynamics and antibody recognition of the meningococcal lipidated outer membrane protein LP2086 in micellar solution. Biochim. Biophys. Acta 1798, 87-93. doi: 10.1016/j.bbamem.2009.09.021

Masignani, V., Comanducci, M., Giuliani, M. M., Bambini, S., Adu-Bobie, J., Arico, B., et al. (2003). Vaccination against Neisseria meningitidis using three variants of the lipoprotein GNA1870. J. Exp. Med. 197, 789-799.

May, K. L., Lehman, K. M., Mitchell, A. M., and Grabowicz, M. (2019). A stress response monitoring lipoprotein trafficking to the outer membrane. mBio 10:e00618-19. doi: 10.1128/mBio.00618-19

McGuinness, B. T., Clarke, I. N., Lambden, P. R., Barlow, A. K., Poolman, J. T., Jones, D. M., et al. (1991). Point mutation in meningococcal porA gene associated with increased endemic disease. Lancet 337, 514-517. doi: 10.1016/ 0140-6736(91)91297-8

McNeil, L. K., Donald, R. G. K., Gribenko, A., French, R., Lambert, N., Harris, S. L., et al. (2018). Predicting the Susceptibility of meningococcal serogroup B isolates to bactericidal antibodies elicited by bivalent rLP2086, a novel prophylactic vaccine. $m$ Bio 9:e00036-18. doi: 10.1128/mBio.00036-18 
Mehr, I. J., and Seifert, H. S. (1998). Differential roles of homologous recombination pathways in Neisseria gonorrhoeae pilin antigenic variation, DNA transformation and DNA repair. Mol. Microbiol. 30, 697-710. doi: 10. 1046/j.1365-2958.1998.01089.x

Mori, H., Araki, M., Hikita, C., Tagaya, M., and Mizushima, S. (1997). The hydrophobic region of signal peptides is involved in the interaction with membrane-bound SecA. Biochim. Biophys. Acta 1326, 23-36. doi: 10.1016/ s0005-2736(97)00004-7

Newcombe, J., Mendum, T. A., Ren, C. P., and Mcfadden, J. (2014). Identification of the immunoproteome of the meningococcus by cell surface immunoprecipitation and MS. Microbiology 160, 429-438. doi: 10.1099/mic.0. 071829-0

Oliver, K. J., Reddin, K. M., Bracegirdle, P., Hudson, M. J., Borrow, R., Feavers, I. M., et al. (2002). Neisseria lactamica protects against experimental meningococcal infection. Infect. Immun. 70, 3621-3626. doi: 10.1128/iai.70.7. 3621-3626.2002

Oriente, F., Scarlato, V., and Delany, I. (2010). Expression of factor H binding protein of meningococcus responds to oxygen limitation through a dedicated FNR-regulated promoter. J. Bacteriol. 192, 691-701. doi: 10.1128/JB.01308-09

Ostberg, K. L., Derocco, A. J., Mistry, S. D., Dickinson, M. K., and Cornelissen, C. N. (2013). Conserved regions of gonococcal TbpB are critical for surface exposure and transferrin iron utilization. Infect. Immun. 81, 3442-3450. doi: 10.1128/IAI.00280-13

Pace, D., and Pollard, A. J. (2007). Meningococcal A, C, Y and W-135 polysaccharide-protein conjugate vaccines. Arch. Dis. Child 92, 909-915. doi: 10.1136/adc.2006.111500

Rahman, M. M., Kolli, V. S., Kahler, C. M., Shih, G., Stephens, D. S., and Carlson, R. W. (2000). The membrane phospholipids of Neisseria meningitidis and Neisseria gonorrhoeae as characterized by fast atom bombardment mass spectrometry. Microbiology 146(Pt 8), 1901-1911. doi: 10.1099/00221287-1468-1901

Rappuoli, R., Pizza, M., Masignani, V., and Vadivelu-Pechai, K. (2018). Meningococcal B vaccine: the journey from research to real world experience. Expert Rev. Vacc. 17, 1111-1121. doi: 10.1080/14760584.2018.1547637

Sanders, H., Brehony, C., Maiden, M. C., Vipond, C., and Feavers, I. M. (2012). The effect of iron availability on transcription of the Neisseria meningitidis fHbp gene varies among clonal complexes. Microbiology 158, 869-876. doi: 10.1099/mic.0.054957-0

Sankaran, K., and Wu, H. C. (1994). Lipid modification of bacterial prolipoprotein. Transfer of diacylglyceryl moiety from phosphatidylglycerol. J. Biol. Chem. 269, 19701-19706.

Schneider, C. A., Rasband, W. S., and Eliceiri, K. W. (2012). NIH Image to ImageJ: 25 years of image analysis. Nat. Methods 9, 671-675. doi: 10.1038/nmeth.2089

Schneider, M. C., Exley, R. M., Chan, H., Feavers, I., Kang, Y. H., Sim, R. B., et al. (2006). Functional significance of factor $\mathrm{H}$ binding to Neisseria meningitidis. J. Immunol. 176, 7566-7575. doi: 10.4049/jimmunol.176.12. 7566

Schneider, M. C., Prosser, B. E., Caesar, J. J., Kugelberg, E., Li, S., Zhang, Q., et al. (2009). Neisseria meningitidis recruits factor $\mathrm{H}$ using protein mimicry of host carbohydrates. Nature 458, 890-893. doi: 10.1038/nature07769

Spidlen, J., Breuer, K., Rosenberg, C., Kotecha, N., and Brinkman, R. R. (2012). FlowRepository: a resource of annotated flow cytometry datasets associated with peer-reviewed publications. Cytometry A 81, 727-731. doi: 10.1002/cyto. a. 22106

Steiner, D., Forrer, P., Stumpp, M. T., and Pluckthun, A. (2006). Signal sequences directing cotranslational translocation expand the range of proteins amenable to phage display. Nat. Biotechnol. 24, 823-831. doi: 10.1038/nbt1218

Tettelin, H., Saunders, N. J., Heidelberg, J., Jeffries, A. C., Nelson, K. E., Eisen, J. A., et al. (2000). Complete genome sequence of Neisseria meningitidis serogroup B strain MC58. Science 287, 1809-1815. doi: 10.1126/science.287.5459.1809

Tokuda, H. (2009). Biogenesis of outer membranes in Gram-negative bacteria. Biosci. Biotechnol. Biochem. 73, 465-473. doi: 10.1271/bbb.80778

Tokunaga, M., Tokunaga, H., and Wu, H. C. (1982). Post-translational modification and processing of Escherichia coli prolipoprotein in vitro. Proc. Natl. Acad. Sci. U.S.A. 79, 2255-2259. doi: 10.1073/pnas.79.7.2255

Vernikos, G., and Medini, D. (2014). Bexsero(R) chronicle. Pathog. Glob. Health 108, 305-316. doi: 10.1179/2047773214Y.0000000162

Vlasuk, G. P., Inouye, S., and Inouye, M. (1984). Effects of replacing serine and threonine residues within the signal peptide on the secretion of the major outer membrane lipoprotein of Escherichia coli. J. Biol. Chem. 259, 6195-6200.

Vogeley, L., El Arnaout, T., Bailey, J., Stansfeld, P. J., Boland, C., and Caffrey, M. (2016). Structural basis of lipoprotein signal peptidase II action and inhibition by the antibiotic globomycin. Science 351, 876-880. doi: 10.1126/science. aad 3747

Vu, D. M., Pajon, R., Reason, D. C., and Granoff, D. M. (2012). A broadly cross-reactive monoclonal antibody against an epitope on the n-terminus of meningococcal fHbp. Sci. Rep. 2:341. doi: 10.1038/srep00341

Welsch, J. A., Ram, S., Koeberling, O., and Granoff, D. M. (2008). Complementdependent synergistic bactericidal activity of antibodies against factor H-binding protein, a sparsely distributed meningococcal vaccine antigen. J. Infect. Dis. 197, 1053-1061. doi: 10.1086/528994

Welsch, J. A., Rossi, R., Comanducci, M., and Granoff, D. M. (2004). Protective activity of monoclonal antibodies to genome-derived neisserial antigen 1870, a Neisseria meningitidis candidate vaccine. J. Immunol. 172, 5606-5615. doi: 10.4049/jimmunol.172.9.5606

Zhang, Q., Li, Y., and Tang, C. M. (2010). The role of the exopolyphosphatase PPX in avoidance by Neisseria meningitidis of complement-mediated killing. J. Biol. Chem. 285, 34259-34268. doi: 10.1074/jbc.M110.154393

Zlotnick, G. W., Jones, T. R., Liberator, P., Hao, L., Harris, S., Mcneil, L. K., et al. (2015). The discovery and development of a novel vaccine to protect against Neisseria meningitidis Serogroup B Disease. Hum. Vacc. Immunother. 11, 5-13. doi: $10.4161 /$ hv.34293

Conflict of Interest: The authors declare that the research was conducted in the absence of any commercial or financial relationships that could be construed as a potential conflict of interest.

Copyright (c) 2019 da Silva, Karlyshev, Oldfield, Wooldridge, Bayliss, Ryan and Griffin. This is an open-access article distributed under the terms of the Creative Commons Attribution License (CC BY). The use, distribution or reproduction in other forums is permitted, provided the original author(s) and the copyright owner(s) are credited and that the original publication in this journal is cited, in accordance with accepted academic practice. No use, distribution or reproduction is permitted which does not comply with these terms. 